\title{
UNC LASSIFIED
}

\section{AECU-4437}

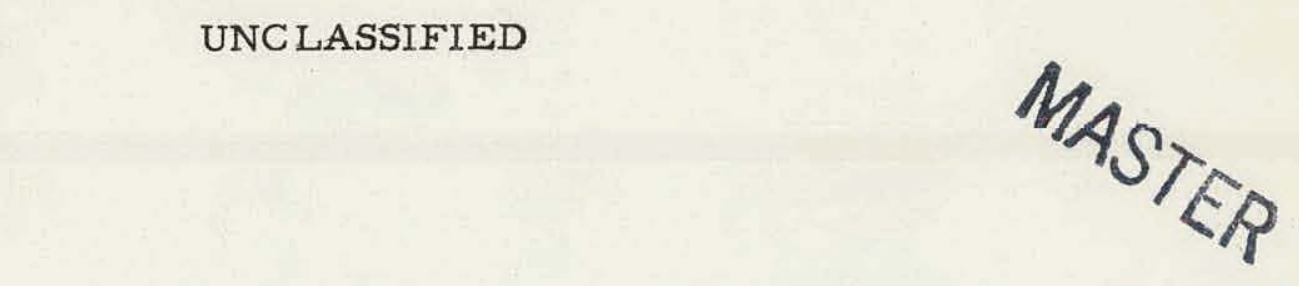

ANNUAL REPORT

\author{
L. Madansky \\ M. Ramaswamy \\ Department of Physics \\ The Johns Hopkins University \\ Baltimore, Maryland
}

Part A: Studies in K-Capture Positron Branching Ratios

Part B: Search for a Low-Lying $0^{+}$State in Gallium-68

AEC Contract AT $(30-1)-2028$, Task B

August 1959

UNCLASSIF IED 


\section{DISCLAIMER}

This report was prepared as an account of work sponsored by an agency of the United States Government. Neither the United States Government nor any agency Thereof, nor any of their employees, makes any warranty, express or implied, or assumes any legal liability or responsibility for the accuracy, completeness, or usefulness of any information, apparatus, product, or process disclosed, or represents that its use would not infringe privately owned rights. Reference herein to any specific commercial product, process, or service by trade name, trademark, manufacturer, or otherwise does not necessarily constitute or imply its endorsement, recommendation, or favoring by the United States Government or any agency thereof. The views and opinions of authors expressed herein do not necessarily state or reflect those of the United States Government or any agency thereof. 


\section{DISCLAIMER}

Portions of this document may be illegible in electronic image products. Images are produced from the best available original document. 


\section{ABSTRACT}

$\mathrm{K}$-capture to positron branching ratios have been measured in the decay of $\mathrm{Na}^{22}$, $\mathrm{Co}^{58}$ and $\mathrm{Ga}^{68}$ - all pure Gamow-Teller emitters, using coincidence scintillation spectrometer techniques. The measured values are $0.105 \pm 0.004$ for $\mathrm{Na}^{22}, 5.08 \pm 0.17$ for $\mathrm{Co}^{58}$, and $1.28 \pm 0.12$ and $0.10 \pm$ 0.02 for $\mathrm{Ga}^{68}$. From these the Fierz interference term is computed to be $b=-0.004 \pm 0.012,-0.004 \pm 0.14,-0.03 \pm 0.02$ and $+0.03 \pm 0.01$ respec. tively. These results indicate that the Fierz interference in Gamow-Teller interaction is very smáll.

The decay of 270 day $\mathrm{Ge}^{68}$ was investigated in equilibrium with Ga ${ }^{68}$ to Look for-a possible low-lying $\mathrm{O}^{+}$level in Ga6m using $X-r a y \cdot X-r a y$ and $X-r a y$

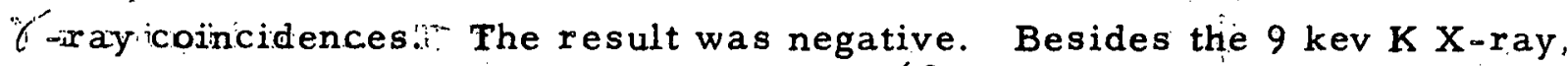
the $1.07 \mathrm{Mev}$ gamma $\mathrm{ray}$ in the decay of $\mathrm{Ga}^{68}$ and annihilation radiation, no other gamma rays were detected $(<8 \mathrm{o} / 0$ of $1.07 \mathrm{Mev}$ gamma ray). The number of positrons per $1.07 \mathrm{Mev} \gamma$-quantum was determinied as $19.47+$ 2. 10. The ratio of positrons to the $1.07 \mathrm{Mev}$ level and ground state of $\mathrm{Zn}^{68}$ was found to be $(1.76+0.22) \times 10^{-2}$.

Studies with a $\mathrm{Ge}^{68}$ source chemically separated from Ga ${ }^{68}$ showed no gamma rays ( $<1 \%$ \% of total $\mathrm{Ge} \mathrm{e}^{68}$ decays). By following the growth of annihilation radiation, an upper limit of $0.4 \% / 0$ per decay could be set on the amount of positron emission by $\mathrm{Ge}^{68}$.

A search for an electric monopole (EO) transition from the 2. $3 \mathrm{Mev}$ level to the $\mathrm{O}^{+}$ground state of $\mathrm{Zn}^{68}$. was made using plastic scintillators and an anti-coincidence arrangement. An upper limit of $(5 \pm 25) \times 10^{-9}$ conversion electrons per decay of $\mathrm{Ga}^{68}$ could be set. This is interpreted as evidence against a possible $\mathrm{O}^{+}$spin assignment for the $2.3 \mathrm{Mev}$ level, but the interpretation is not conclusive.

A brief review is presented regarding, the status of Fierz interference in beta-decay as revealed by present studies and other-experiments. 
General Introduction

a) The Interaction in Beta-Decay:

The central problem in the theory of beta-decay has been the determination of the nature of the interaction responsible for this decay. In general the interaction can be a linear combination of five types, namely scalar (S), vector (V), tensor (T), axial vector (A), and pseudoscalar (P), all satisfying the requirement of relativistic invariance. Beta-decay can be classified as allowed or forbidden depending on the change in angular momenta and parities of the nuclear states involved. The selection rules permit a further distinction between transitions as Fermi or Gamow-Teller. The selection rules are:

$$
\text { Allowed } \Delta \mathrm{J}=0 \quad \text { Fermi }
$$

$$
\begin{aligned}
& \text { No } \\
& \Delta \mathrm{J}=0,+1 \quad \text { Gamow-Teller } \\
& \text { No } \quad 0 \rightarrow 0 \\
& \text { First forbidden } \Delta \mathrm{J}=0 ; \pm 1, \pm 2 \quad \text { Gamow-Teller } \\
& \text { Yes } \\
& 0, \pm 1 \quad \text { Fermi }
\end{aligned}
$$

and so on.

The Fermi transitions involve only the interactions $S$ and $V$, and the interactions $A$ and $T$ characterize Gamow-Teller transitions. A transition allowed by both types of selection rules should therefore involve:S, V, A, T and perhaps $P$. There is strong evidence that the $P$ interaction is unimportant. The fact that transitions obeying both kinds of selection $r$ ules are ob.served indicates that the betainteraction is an admixture of both. Fermi and Gamow-Teller types. It remains to determine the ratio of these interaction strengths. A study of the angular correlation between the electron and the neutrino in an allowed pure transition can be used to distinguish: which of the interactions $S$ or $V$, or $A$ or $T$ is predominant. It is now established from such experiments that the Fermi interaction is mostly $V$ and the Gamow. Teller interaction mostly $A$. The neutron decay (mixed transition) combined with the $\mathrm{O}^{14}$ decay (pure Fermi transition) leads to the determination of the relative strengths of Fermi and Gamow-Teller interactions. The recent Russian measurement of $11.7 \pm 0.4 \mathrm{~min}$. for the half life of the neutron 
leads to $\left(\mathrm{C}_{\mathrm{GT}} / \mathrm{C}_{\mathrm{F}}\right)^{2}=1.42 \pm 0.08$

Considering only pure transitions, Fermi or Gamow-Teller, one can. expect interference between the two types $S$ and $V$, or $A$ and $T$. The pos sible existence of such terms.was. first pointed out by Fier ${ }^{2}$ and hence these terms are called.Fierz interference terms. It is the principal objective of the present work to make an estimate of this effect in Gamow-Teller transitions. Such interference is possible in the electron-neutrino angular correlation gxpression, but because of the difficulties involved in such experiments these terms are often neglected. Intexference between $A$ and $V$ in a mixed transition can also occur, but we will not concern ourselves with this here, nor will we treat forbidden transitions.

b) Fierz Interference

The general expression for the energy distribution of electrons (posi. trons) in an allowed transition can be witten as ${ }^{3}$

$$
N(w) d w=\left[2 \pi^{3}\right]^{-1} p w(w-w)^{2} F(z, w) \xi(1 \pm 2 . b / w) d w
$$

where

$$
\begin{aligned}
\xi= & \left.\iint 1\right|^{2}\left[k^{-2}\left(\left|C_{S}\right|^{2}+\left|C_{S}^{\prime}\right|^{2}\right)+\left(\left|C_{V}\right|^{2}+\left|C_{V}^{\prime}\right|^{2}\right)\right]+ \\
& \left.1 \int \sigma\right|^{2}\left(\left|C_{A}\right|^{2}+\left|C_{A}^{\prime}\right|^{2}+\left|C_{T}\right|^{2}+\left|C_{T}^{\prime}\right|^{2}\right)
\end{aligned}
$$

and

$$
\xi b= \pm \gamma\left[\left.1 \int 1\right|^{2} \operatorname{Re}\left\{k^{-1}\left(C_{S} C_{V}^{*}+C_{s}^{\prime} C_{V}^{\prime *}\right)\right\}+\left|\int \sigma\right|^{2} \operatorname{Re}\left\{C_{C_{C}} C_{T^{*}}^{*}+\right.\right.
$$
$\left.\left.\left.C_{A}^{\prime} C_{T}^{\prime *}\right)\right\}\right]$

Here the + sign refers to elect $r$ on and - to positron emission. The other symbols are explained as follows:

$\mathrm{pW}\left(\mathrm{W}_{\mathrm{o}}-\mathrm{W}\right)^{2}$ is the statistical weight factor: which determines, in the absence of the coulomb field, the sharing of energy between the electron and the neutrino.

$F(Z, W)$ is the coulomb field factor which represents the effect of nuclear charge on the emitted electron.

$p$ is the momentum of the electron. 
$W$ is the energy of the electron in relativistic units.

$W_{0}$ is the maximum energy of the electron or positron

where $\quad k=\int_{\int}^{1} 1 / \sqrt{ } \beta$

$\int \beta=$ the vector matrix element

$k=1$ only if the motion of the nucleons is non-relativistic, since in this case $\beta=\gamma_{4}=1$.

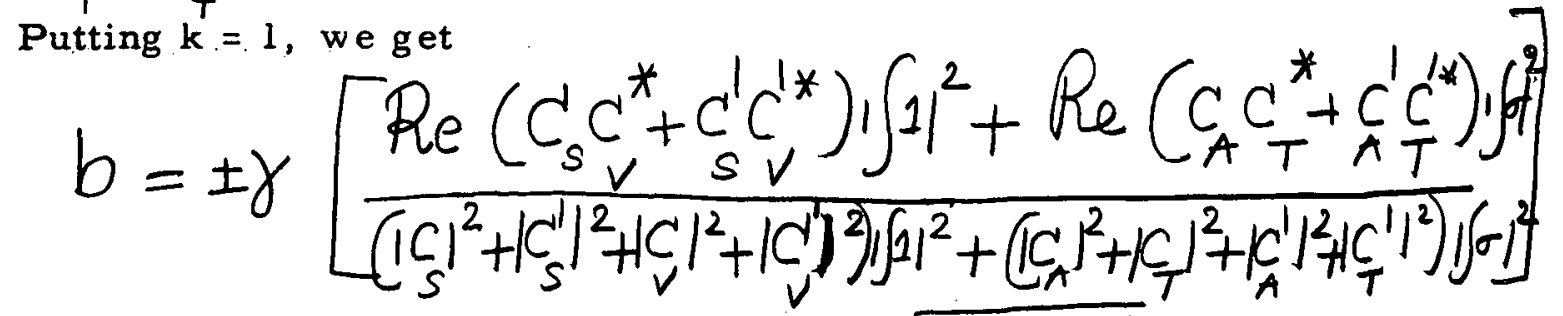

is called the Fierz interference term. Here $\gamma=\sqrt{1-(\alpha z)^{2}} \cong 1$ represents the screening effect due to the atomic electrons.

$C_{i}=S, V, A_{j}, T=$ is the coupling constant for parity conservidig inter action

$C_{i}^{\prime}=S, V, A ; T=$ is the coupling constant for parity non-conserving interaction.

The complex conjugation on the coupling constants represents the possibility of time reversal invariance in the beta-decay process.

An immediate consequence of $b \neq 0$ is that the spectral shape of an allowed transition will deviate from the statistical shape because of the in. verse dependence on $W$ through $b$. One way of seeing this deviation experimentally is to plot the form factor $N(W) /\left[F(z, W) p W\left(W_{0}-W\right)^{2}\right]_{a s}$ a function of $W$. From this kind of analysis the limits set on $b_{G T}$ are $-0.09 \leqslant b_{G T} \leqslant 0.20$. Because of the weak dependence on W-such deviations are rather hard to detect. Further the analysis has so far been generally restricted to Gamow Teller transitions only. Recently, Danie $1^{4}$ has applied this method to estimate the Fiexz term in the decay of $\mathrm{N}^{13}\left(1 / 2^{-}-1 / 2^{-}\right)$. He obtained $b_{F}=$ 0.14 using the $\mathrm{O}^{14}$ ft value to evaluate the Fermi part of the matrix element. Integrating expression (1) over the allowed spectrum, we obtain.

$$
2 \pi^{3}(S t)^{-1} \ln 2=\xi+\xi b\left\langle W^{-1}\right\rangle
$$


Wo

where $\mathrm{f}=\int_{1} F(z, W)(W, W)^{2} p W d W$ is the so-called Fermi function, and $\left\langle W^{-1}\right\rangle=f^{-1} \int_{1}^{W_{0}} F(z, W) P(W-W)^{2} d W$ is the expectation value of $W^{-1}$ over the allowed spectrum.

Thus a consequence of $b \neq 0$ is that the $\mathrm{ft}$ values will depend on $\mathrm{w}^{-1}$. From a plot of $2 \pi^{3}\left[\mathrm{ft}\left|\left(\left.1\right|^{2}\right]^{-1} \ln 2 \mathrm{vs} .2 \gamma_{1} \int 1\right|^{2}\left\langle W^{-1}\right\rangle\right.$ which should give a straight line provided $k=1$ and the matrix elements remain the same, Ger. hart ${ }^{3}$ finds from an analysis of data for $0 \quad 0$, No (Fermi) transitions $-\infty 0^{14}$, $\mathrm{Al}^{26}$ and $\mathrm{Cl}^{3.4}$, that

$$
b_{F}=\gamma \frac{\operatorname{Re}\left({ }_{C} C_{s}{ }^{*}+C_{s}^{\prime} C_{v}^{\prime}\right)}{\left(\left.C_{s}\right|^{2}+\left|C_{s}^{\prime}\right|^{2}+\left|C_{v}\right|^{2}+\left|C_{v}\right|^{2}\right.}=0.00 \pm 0.12
$$

the chief uncertainty being due to the assumption regarding $k$. (Recently Altman and MacDonald ${ }^{5}$ have considered the effect of coulomb and relativis. tic corrections to the evaluation of the. Fier $z$ term and conclude that the corrections are within experimental uncertainties.) The matrix elements were evaluated $b$ y Gerhart on the basis of charge independence of nuclear forces.

Another fruitful approach for the evaluation of $b$ has been the method of $\mathrm{K}$-capture to positron branching ratios first exploited by Sherr and Miller 6 . In the following section we will describe the infolmation that can be dexived from a study of $\mathrm{K} / \beta+$ ratios and in particular about the Fierz term.

\section{c) K-Capture Positron Branching Ratios}

The study of the shapes of beta-spectra together with the ft values and the shell model (to determine parities) has been very useful in classifying transitions as to the order of forbiddenness. When, how iver, between two nuclear states enough energy is available for both $K$-capture and positron emission, a useful quantity that can be measured is the K-capture positron branching ratio. In fact it was one of the early triumphs of the Fermi theory of beta-decay that the K-capture mode of decay was observed as predicted. A measurement of $K / \beta^{t}$ ratio can be used to find the energy difference be tween two nuclear states if it is known otherwise that the transition is allowed. However, it is observed ${ }^{7}$ that all allowed shape transitions (most first-for bidden transitions) have allowed branching ratios also. Thus it is not possible 
5

to determine whether an allowed shape transition is indeed allowed, wi thou a knowledge of the parity change. However, the $\mathrm{K} / \beta+$ ratio does show a detectable change for unique first forbidden and higher transitions intensifled with increasing order of forbiddenness ${ }^{8}$. These latter transitions can. probably be much more easily identified on the basis of the shape of the positron spectrum and lifetime. In such cases $\mathrm{K} / \beta+$ ratios can only serve as an additional check on the assignment. However, the chief virtue of measurement of the $\mathrm{K} / \beta+$ ratio for supposedly pure transitions is that it. lends itself to the estimation of small-order effects in beta-decay such as the Fiery term. Consider a pure transition, say a Gamow -Teller transtron. Then for this transition the probability for positron emission is

$$
P_{+}=\frac{1}{2 \pi} \int_{1}^{W_{0}} f(z, w) p w(w-w)^{2} d W \xi(1-2 b / w)
$$

where the various quantities have already been defined (see page 2). (Note that the terms involving $C_{S}$ and $C_{V}$ are set equal to zero.)

The probability for $\mathrm{K}$-capture to the same state can be written as

$$
P_{k}=\frac{1}{4 \pi^{2}}\left(w_{0}+W_{k}\right)^{2} g_{k}^{2}(R) \xi(1+2 b)
$$

where $g_{k}^{2}(R)=\frac{1+\gamma}{2 \Gamma(2 \gamma+1)} R^{2 \gamma-2}\left(2 \alpha z_{\text {eff }}\right)^{2 \gamma+1}$ is the Dirac radial function.

$$
\begin{aligned}
& \mathrm{w}_{\mathrm{o}}=\text { total energy available for the transition in } \mathrm{m}_{\mathrm{o}} \mathrm{c}^{2} \text { units, } \\
& \mathrm{w}_{\mathrm{K}}=\gamma \simeq \sqrt{1-\alpha^{2} z^{2}}
\end{aligned}
$$

So that the ratio of $\mathrm{K}$-capture to positron emission becomes

$$
P_{K} / P_{+}=\frac{\left(1 / 4 \pi^{2}\right)\left(W+W_{k}\right)^{2} g_{K}^{2}(R) \xi(1+2 b)}{\left(1 / 2 \pi^{3}\right) \int_{1}^{W_{0}} F(z, W) P W(W-W)^{2} d W \xi(1-2 b / W)}=R
$$

If the Fiery interference term. were zero, then putting $b=0$, we get

$$
\left(P_{k} / P_{+}\right)=\frac{\left(1 / 4 \pi^{2}\right)\left(W_{0}+W_{k}\right)^{2} g_{k}^{2}(R)}{\left(1 / 2 \pi^{3}\right) \int_{\text {Dividing (2) by (1), we obtain }}^{W_{0}} F(z, w) p W(W-W)^{2} d w}=R_{0}
$$

$$
R / R_{0}=\frac{1+2 b}{1-2 b(w-1)]}
$$

where $\mathrm{W}^{-1}$ has already been defined.

$$
\therefore b=\frac{R / R_{0}-1}{2\left[1+R / R_{0}\left\langle W^{-1}\right\rangle\right]}
$$

$09 \% \quad 027$ 
Thus a measurement of $R$ can be used to evaluate $b$. It should be noted that the matrix elements cancel out in the ratio.

Before comparing the theoretical $\mathrm{K} / \beta+$ ratio with the observed value, correction for the finite size of the nucleus and screening of the positron and the bound $K$ electron have to be made. Further, if the measured quantity is the total electron-capture, then correction for capture from higher shells has to be made to obtain the $\mathrm{K}$-capture alone.

For allowed transitions the finite size correction has been shown to be negligible ${ }^{7}$. The screening correction, on the other hand, is not insignifi. cant. Recently Perlman, Welker and Wolfsberg ${ }^{9}$ have evaluated the effect of screening on the positron wave function and have given in graphical form the ratio of screened to unscreened values. For most $Z$ values of interest the screening on the $K$-electron is taken into account by putting $Z_{\text {effective }}=Z_{K}$ - 0.3. Zweifel ${ }^{7}$ has evaluated the deviation of the actual $Z_{\text {eff }}$ from this Slater screening. Regarding correction for capture from higher shells. only L. capture is important for most cases of interest. (At high $\mathrm{Z}, \mathrm{M}$ capture also becomes important.) Correction for Lecapture is obtained by using L/K ra= tios given in graphical form by Rose and Jackson 10 .

We have applied the $\mathrm{K} / \beta^{+}$ratio technique for the decays of $\mathrm{Ga}^{68}, \mathrm{Co}^{58}$ and $\mathrm{Na}^{22}$, all pure Gamow - Teller emitters, to obtain the Fierz interference term. Part $\mathrm{Af}_{\mathrm{f}}$ this report contains a description of experiments on each of these nuclei, followed by a summary of available data on Fierz interference in beta-decay as obtained by various methods. Part B of the report deals with some incidental studies on a search for a low olying $\mathrm{O}^{+}$state in $\mathrm{Ga}^{68}$ and a search for electric monopole transition from the $2,3 \mathrm{MeV}$ level in $\mathrm{Zn}^{68}$. 
PART A

1. K Capture Positron Branching Ratios in the Decay of $\mathrm{Ga}^{68}$

a. Introduction

The decay of $68 \mathrm{~min}$ Ga-68 has been studied by Muker ji and Preis. werk ${ }^{11}$ and $x$ ecently by Crasemann et al. ${ }^{12}$. The decay was found to consist of two modes, the predominant mode $(\$ 87 \mathrm{o} / 0)$ being decay to the ground state of $\mathrm{Zn}-6.8$ by positron emission and electron capture, and the other branch leading to the first excited state at $1.07 \mathrm{Mev}$ by positron emission and electron capture followed by a gamma-ray of about 1.1 Mev. The energies of the positron groups leading to the ground state and the first excited state were measured by means of a magnetic spectrometer to be $1.88 \mathrm{Mev} \pm 0 .$, and 0.77 Mev respectively by Mukerji and Preiswerk ${ }^{11}$ and found to be $1 . \overline{9} \pm \pm 0.05$ and $0.92 \mathrm{Mev}$ by Crasemann ${ }^{12}$. More recently Daniel 13 has measured the endpoint of the high energy positron group to be $1.88 \pm 0.02 \mathrm{Mev}$. The ratio of the intensities of the low energy positron group to the high energy group was found by Creasemann to be $(4.1 \pm 1.4) \times 10^{-2}$. No other gamma-rays were obsexved, and conversion electrons were absent.

The $\log$ ft values for the positron decays are 5.3. (to the ground state) and 5.2 (to the $1.07 \mathrm{Mev}$ level). The spin of the ground state of even even $\mathrm{Zn}-68$ is $0^{+}$and that of the first excited state $2^{+}$from systematics of eveneven nuclei ${ }^{14}$. These spins together with the allowed $\log$ ft values make $1^{+}$a most likely assignment for the Ga-68 ground state. This assignment has been confirmed by a recent direct measurement of spin by Hubbs et al. ${ }^{15}$. It fol. lows then that the positron decays are pure Gamow-Teller transitions $(\Delta \mathrm{J}=1$, No). Hence it was thought worthwhile to measure the $K / \beta+$ ratios to obtain an estimate of the Fierz term. Incidental to these measurements, other quantities of interest were also obtained.

b. Source Preparation

Since the decay of 270-day Ge-68 leads to the 68 minute Ga-68, a source of Ge-68 was produc ed a follows. Zinc was electroplated onto a copper backing and was bombarded for 96 microampexe-hours with $30 \mathrm{Mev}$ alpha-particles at the cyclotron of the Department of Terrestrial Magnetism, Washington, D. C. (through the courtesy of Drs. Heydenburg and Temmer). 23 weeks after the bombardment the zinc layer on the copper backing was 
dissolved in a few cc of conc. $\mathrm{HCl}$ containing Ge carrier. $\mathrm{GeCl}_{4}$ was dis tilled onto a receiver. $\mathrm{GeS}_{2}$ was precipitated by saturating with $\mathrm{H}_{2} \mathrm{~S}$, then washed and dissolved in $\mathrm{NH}_{4} \mathrm{OH}$. Sources were prepared by evaporating drops of this solution onto a formvar backing. Measurements were made on $G a-68$ in equilibxium with $G e-68$.

c. $\mathrm{K} / \beta+$ Ratios

Description of the method: The decay scheme as given by Crasemann et al 12 is shown schematically in Fig. 1. Since our measurements were made on Ga -68 in equilibrium with Ge-68, the numbers of the se two nuclei decaying per unit time are equal. If $f_{\beta}^{1}+$ and $f_{E}^{1}$ denote the fraction of positrons and electron-captures to the first excited state at $1.07 \mathrm{Mev}$ and $f_{\beta+}^{0}$ and $f_{\epsilon}^{0}$ denote corresponding quantities to the $0^{+}$ground state, then we have $\epsilon$

$$
f_{\beta}{ }^{1}+f_{\epsilon}^{1}+f_{\beta+}^{0}+f_{\epsilon}^{0}=1
$$

Now consider the arrangement shown schematically in Fig. 2. As is indicated in the figure, counter $S$ I and II detect annihilation radiation, counter III 1.07 Mev gamma-ray, and counter IV, $\mathrm{K} \mathbf{X}-\mathbf{r a y}$

For the singles counting rate we have

$$
\begin{aligned}
& N_{0.5}^{I}=2 N_{0} E^{I}\left(f_{\beta}^{1}+f_{\beta+}^{0}\right)=a \\
& N_{1.07}^{\text {III }}=N_{0} E^{\text {III }}\left(f_{\beta^{+}}^{1}+f_{\epsilon}^{1}\right)=b \\
& N_{x}^{\text {IV }}=N_{0} f_{y} E^{\text {II }}\left(f_{\epsilon}^{1}+f_{\epsilon}^{0}+1\right) /(1+L / k)=c
\end{aligned}
$$

where the superscripts denote the counters and the subscripts denote the radiations being measured,

$$
\begin{aligned}
& E=\text { efficiency of the counter } \\
& N_{0}=\text { the source strength } \\
& f_{y}=\text { the } K \text { fluorescence yield } \\
& \text { and L/K the ratio of } L \text { to } K \text { captures. }
\end{aligned}
$$

For the coincidences, we have

$$
\begin{aligned}
& N_{0.5,0.5}^{I, I}=2 N_{0} E^{a}\left(f_{\beta}{ }^{1}+f_{\beta+}^{0}\right)=\alpha \\
& N_{0,5,1.07}^{I, \text { I }}=2 N_{0} E^{I} E^{I I, \Pi} f_{\beta^{+}}^{1}=2 N_{0} E^{\frac{V}{I I}} f_{\beta^{+}}^{1}=\beta \\
& N_{x, 1.07}^{\text {II II }}=N_{0} E^{\text {II }} E^{\text {III }^{\prime}} f_{\epsilon}^{1} /(1+L / K)^{\beta}=\gamma
\end{aligned}
$$


Where $E$ is the combined efficiency of counters I, II for annihilation radiation $\mathbf{E}^{\mathrm{III} !}=$ efficiency of the 1.07 counter without absorber (used to annihilate positrons).

The preceding analysis is based on the following assumptions:

a) Conversion of the $1.07 \mathrm{MeV}$ gamma-ray is negligible

b) Contribution to $\mathrm{X}$ rays due to pure electron-capturing 12-day $\mathrm{Ge}-71$ is negligible

c) No positrons are emitted by Ge- -68

d) The contribution to annihilation radiation due to pairs from 1.07 MeV gamma ray is negligible

e) Compton background of 1.07 gamma $\times$ ray has been subtracted from the 0.511 photo-peak

f) The decay scheme is essentially correct.

With these assumptions and with the help of the preceding relations, we obtain

$$
\begin{aligned}
& f_{E}^{1 / f_{\beta^{+}}^{1}}=\frac{a b}{\alpha \beta} \frac{E^{a}}{E^{I}} \frac{E^{I}}{E^{I I}}-1=\left[\frac{a b}{\alpha \beta}\right]_{G a^{68}}\left[\frac{\alpha \beta}{a b}\right]_{N a^{22}} 1 \cdot 11-1 \\
& f_{\beta^{+}}^{1} / f_{\beta^{+}}^{0}=\frac{a}{b}\left[1+\frac{f_{\epsilon}^{1} / f_{\beta^{+}}^{1}}{1}\left[\frac{\left(2 E^{I}\right.}{E^{I}}\right)-1\right.
\end{aligned}
$$

and

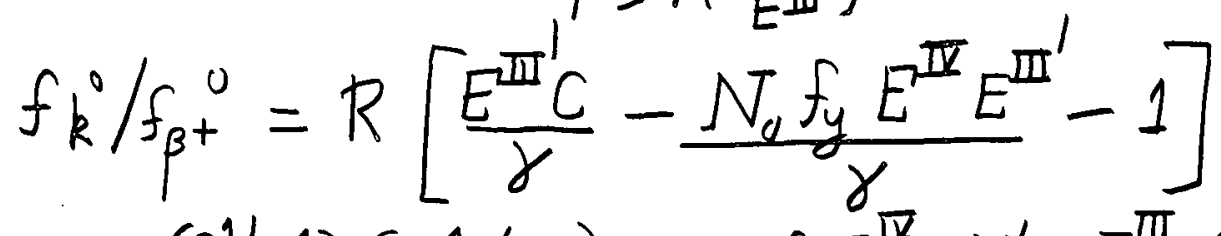

where $R=\left(f_{k}^{1} / f_{\beta^{+}}^{1}\right)\left(f_{\beta^{+}}^{1} / f_{\beta+}^{0}\right)$ and $f_{y} E^{\bar{\nabla}}=(\gamma / b) \frac{E^{\text {III }}}{E^{\text {II }}}\left(1+1 /\left(f_{k}^{1} / f_{\beta^{+}}^{1}\right)\right)$ In these equations the unknowns to be found are the various efficiencies and $N_{0}$, the source strength. We can assume with negligible exror that. $\mathbf{E}^{\text {III }}=$ $E^{I I I^{\prime}}$. To find the various efficiencies, we replace $\mathrm{Ga}^{6} \&_{\text {by }} \mathrm{Na}^{22}$ which has similar and known decay scheme. However; in using $\mathrm{Na}^{22}$, two corrections are necessary. First is the difference in range of Lucite of $\mathrm{Na}^{22}$ positrons ( $0.540 \mathrm{MeV}$ ) and $\mathrm{Ga}^{68}$ positrons ( N1.9 MeV). This will affect the solid angle of the counters for annihilation radiation. Secondly, the efficiency of $1 \frac{1}{2} " \times 1 " \mathrm{NaI}(\mathrm{T})$ crystal for detecting the $1.28 \mathrm{MeV}$ gamma $\mathrm{ray}$ of $\mathrm{Na}^{22}$ is slighlly luwer than that for detecting the $1: 07 \mathrm{MeV}$ gamma ray of Ga ${ }^{68}: \mathrm{N}_{0}$ can in principle be determined by making a chemical separation of Ga from Ge and studying the $K X$-rays as a function of time. The extrapolation to zero time would then give $\mathbb{N}_{0}$, if the fluorescence yield and the $\mathrm{L} / \mathrm{K}$ capture 
ratio are assumed. This procedure is, how ever, subject to two uncertainties: (a) absorption in the source itself due to softness of the X-rays, and (b) reckoning zero time. An approximate value of $N_{0}$ is given by $N_{0} \simeq 1.15 \mathrm{a} / 2 \mathrm{E}^{I}$, if it be assumed that the positrons account for $87 \mathrm{o} / \mathrm{o}$ of the transitions.

\section{d. Experimental}

The source was placed in a lucite well of $6 \mathrm{~mm}$ thickness all round. This ensured the complete stopping of the positrons. The source plus ab. sorber assembly was mounted on a lucite pillar which could be rotated about an axis. Fig. 2 shows the experimental set-up, which has already been de scribed. Counters 1 and 2 employ $2^{\prime \prime} \times 2$. $\times 2$ " NaI(T1) and counter 3 a $1 \frac{1}{2} "$ $x$ I" NaI(Tl) crystal mounted on DuMont6292 photomultiplier tubes. Counter 4 , which detected the $\mathrm{K} X$-ray, consisted of a thin freshly cleaved $(\sim \mathrm{mm}$ thick and $1 \mathrm{~cm}$ square) NaI crystal mounted directly by means of silicone on another DuMont 629.2 phototube and sealed with an.0.0005" aluminum foil which served as a light reflector. Counters 1 and 2 had resolution (full width at half maximum) of 12 and 16. $/ 0$ respectively for the $0.661 \mathrm{MeV}$ gamma ray of $\mathrm{Cs}-137$. The X-ray counter had resolution of 40 to $60 \% / 0$, depending on the nature of the crystal, for the $9 \mathrm{KeV} \mathrm{K} \mathrm{X-ray.} \mathrm{The} \mathrm{use} \mathrm{of} 2^{\prime \prime}$ cube NaI(Tl) crystals facilitated the evaluation of the Compton contribution of the 1.07 $\mathrm{MeV}$ gamma ray of Ga-68 or $1.28 \mathrm{MeV}$ gamma ray of $\mathrm{Na}-22$ to the $0.511 \mathrm{MeV}$ photopeak. The use of very thin crystals for the $\mathrm{K} \mathbf{X}$-ray helped to discriminate considerably against higher energy gamma rays.

The outputs from the phototubes were fed to Atomic Model $205 \mathrm{~B}$ pre. amplifiers, and after amplification by Model N-301 non-overloading Hamner amplifiers were analyzed by means of Model 610 single channel analyzers of the Atomic Instrument Company. The outputs from the pulse height analyzer $s$ could be fed to a scaler. For the coincidence measurements the outputs from the pulse height analyzers (whose delays were matched) were fed to a coincidence circuit whose resolving time $2 \tau$ was determined by two independent source methods to be 3.2 microseconds. The experiments which initially were done with single channel analyzers were subsequently repeated with Model $520 . M 20$ channel analyzer of the Atomic Instrument Company. In this case the variable gate in the multi-channel analyzer replaced the coincidence cir cuit. 
The experimental procedure was to measure the singles and coincidences first with $\mathrm{Ga}-68$ and then with $\mathrm{Na}-22$ under identical geometry. In order to eliminate the effects of scattering from the surrounding shield, no shielding was used. Source to detector distances of $l^{\prime \prime}$ to $1 \frac{1}{2}$ "l were used. Whereas the procedure for estimating the counting rates for the annihilation radiation and $1.07 \mathrm{MeV}$ gamma ray were straightforward, the analysis of the $\mathrm{K}$ $\mathrm{X}$-ray presented a problem. It was found that despite the use of very thin crystals, the K X-ray peak was superposed on a rather high background, apparently due to the higher energy gamma rays. In order to obtain the inten sity of the observed $X$-ray, the spectrum was repeated with an 0.01 " aluminum foil which would attenuate the $X-r$ ay but not the high energy gamma rays. By normalizing this spectrum with the spectrum without the absorber beyond the $\mathrm{K}$ X-ray region and subtracting the background, the intensity of the $\mathrm{K} X$ rays could be estimated. It must be mentioned in this connection that the use of non-overloading amplifiers in observing the $\mathrm{K} X$-ray was very essential.

\section{e. Results}

Table I summarizes the results of a typical run. Background correc$t$ ions have been made. With the weak source used $(\sim 1 / 10$ microcurie $)$ and a resolving time of 3 microseconds, the accidental rate was negligible. The corrections mentioned earlier for $\mathrm{Na}-22$ were made as follows: To deter mine the $0.511 \mathrm{MeV}$ solid angle correction, the counting rate in the annihila= tion radiation peak was measured as a function of distance between source and detector. From this the correction corresponding to the actual distance used in the experiment was obtained. This amounted to $(10 \pm 0.5) 0 / 0$. The correction for difference in the efficiency of $1 \frac{1}{2} ! ! \times 1 " N a I$ crystal for detect ing 1.07 MeV gamma ray of Ga-68 and 1.28 MeV gamma ray of $\mathrm{Na}-22$ was computed from the curves of Bell to be $8 \% / 0$. It must be pointed out here that the corrections just cancel out for

The singles gamma spectrum of Ga-68 taken.with the 2 " cube crystal is shown in Fig. 3. Besides the annihilation radiation and the gamma $x$ ay around $1 \mathrm{MeV}$, no other gamma ray was observed $(<8 \mathrm{o} / \mathrm{o}$ of $1.07 \mathrm{Mev}$ gamma rayl. The energy of this gamma ray was found to be $1.067 \pm 0.035$ $\mathrm{MeV}$. Na-22 (0.511 and 1.28 MeV, Cs-137 (0.661 MeV) and Co-60 (1.17 and $1.33 \mathrm{MeV}$ ) served as calibration sources. The number of positrons 
Table I. Summaxy of Data on Ga ${ }^{6.8}$ (For symbols, see text)

$$
\begin{aligned}
& \mathrm{Ge}^{68} \\
& \begin{array}{llr}
\text { a } & 93.7 \pm 3.1 \mathrm{cps} & 154.0 \pm 3.9 \mathrm{cps} \\
\mathrm{b} & 0.78 \pm 0.3 \mathrm{cps} & 32.0 \pm 0.7 \mathrm{cps} \\
\mathrm{c} & 86.5 \pm 2.2 \mathrm{cps} & \\
\alpha & 14.73 \pm 0.50 \mathrm{cps} & 25.17 \pm 0.70 \mathrm{cps} \\
\beta & (6.8 \pm 0.4) \times 10^{-3} & (0.56 \pm 0.02) \\
\gamma & (7.5 \pm 0.4) \times 10^{-3} & \\
\mathrm{E}^{\mathrm{III}}=(\beta / a)_{\mathrm{Na}} & 1.08 \times 1.11=(4.78 \pm 0.18) \times 10^{-3}
\end{array} \\
& \begin{array}{c}
\frac{E^{I I I}}{2 E^{I}}=0.85(b / a) N_{a}=0.22 \pm 0.01 \\
N_{O} \simeq(4520 \pm 200) \mathrm{cps}
\end{array} \\
& f_{y} \cdot E^{I V}=(17.0 \pm 0.9) 10^{-3}
\end{aligned}
$$

$f_{k}^{1} / f_{\beta^{+}}^{1}=1.28 \pm 0.12$

$$
\begin{array}{r}
f_{\beta^{+}}^{1} / f_{\beta^{+}}^{0}=\left(1.76 \pm 0.27 \times 10^{-2}\right. \\
\quad f_{k}^{0} / f_{\beta^{+}}{ }^{0}=0.10 \pm 0.0 .2
\end{array}
$$

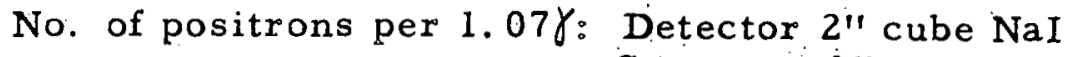

$$
\left.\begin{array}{l}
\mathrm{N}_{1.07 .}=2.08 \mathrm{cps} \\
\mathrm{N}_{0.5}=186.4 \mathrm{cps}
\end{array}\right\} \begin{array}{ll}
\mathrm{Ga}^{j 68} & \mathrm{~N}_{1.28} \\
& \mathrm{~N}_{0.5}
\end{array}
$$

$$
\mathrm{N}_{\beta}+/ \mathrm{N}_{1.07}=\left(\mathrm{N}_{0.5} / \mathrm{N}_{1.07}\right) \mathrm{Ga}\left(\frac{\epsilon_{1.28}}{\epsilon_{0.5}}\right) \frac{1}{2} \quad \text { uncorrected }
$$

Corrections: $\frac{\epsilon_{1.28}}{\epsilon_{1.07}}=0.90:$ solid angle for $0.511=10 \%$

$$
\mathrm{N}_{\beta}+/ \mathrm{N}_{1.07}=(19.47 \pm 2.10)
$$


per $1.07 \mathrm{MeV}$ gamma ray was luund to be $19.47 \pm 2.10$ by comparison with $\mathrm{Na}-22$. An efficiency yatio of $0.90 \pm 0.04$ was assumed for the 1.28 and $1.07 \mathrm{MeV}$ gamma rays. This value is to be compared with $14.4 \pm 1.7$ of Crasemann et al. and 27.3. \pm 3 of Hor en ${ }^{18}$. However; a value of $22 \pm 3$ was also obtained by Hor en from coincidence mea surements. The value for $f_{\beta}+/ f_{\beta} t^{1}$ is in agxeement with the work of Hor en. This quantity is an interesting byproduct, since the ratio of the positron branches is determined without measuring the beta spectrum as such.

\section{f. Discussion.}

Before comparing the experimental results with theory, it is neces. sary to examine some of the assumptions made early in the analysis. The assumption that the conversion of $1.07 \mathrm{MeV}$ gamma ray is negligible is in deed reasonable. For an E2 transition the value of $\alpha_{k}$ is $\sim 10^{-4}$. Both the half-life (12 days) and the abundance of $\mathrm{Zn}^{68}$ from which it was formed make the contribution from Ge $\mathrm{e}^{71}$ negligible. Self excitation is negligible in the weak source employed. It will be shown in Part B of the report, under Ge ${ }^{68}$, that the amount of position emission from Ge 68 is $<0.4 \%$ o. This conclusion. is supported by beta-decay energy systematics of Way and Wood ${ }^{20}$ which pre dicts a decay energy for $\mathrm{Ge}^{68}-\mathrm{Ga}^{68}$ to be $\sim 750 \mathrm{keV}$ or by a plot of $\mathrm{E}_{\beta \beta}$ double beta-decay energy versus neutron number (for Zn.Ge). Linex inter polation gives $500+150 \mathrm{keV}$ for $\mathrm{Ge}^{68}-\mathrm{Ga}^{68}$ decay if it be assumed that the $\mathrm{Ga}^{68}$ - $\mathrm{Zn}^{68}$ decay energy is $2.9 \mathrm{MeV}$. The contribution to annihila. tion radiation due to pairs from the 1.07 gamma ray is certainly negligible 21 . As regards the la st assumption about the correctines of the decay scheme, Horen ${ }^{18}$ has found very recently weak electron capture branchings ( $\sim 0.37 \mathrm{o} / \mathrm{o})$ to higher excited states in $\mathrm{Zn}-68$. This fact will alter somewhat the value for the ground state electron-capture positron branching ratio. But since the errors are already large, our conclusions remain unaffected.

In the general introduction the expression for the Fierz.term was found to be

$$
b=\frac{R / R_{0}-1}{2\left[1+R / R_{0}\left\langle W^{-1}\right\rangle\right]}
$$

in terms of the measured ratio $R_{;}$the theoretical ratio $R_{0}$ ? and $W^{-1}$, the 
average value of $W^{-1}$ over the allowed spectrum. A sufficiently accurate value for $W^{-1}$ can be obtained from an expression due to Gerhart ${ }^{3}$ :

$\left\langle W^{-1}\right\rangle \cong\left(\frac{5}{2 W_{0}}\right)\left[\frac{2 W_{0}^{3} p_{0}+13 W_{0} p_{0}-3\left(4 W_{0}^{2}+1\right) \ln \left(w_{0}+p_{0}\right)}{2 W_{0}^{3} p_{0}-9 w_{0} p_{0}-8 p_{0} / w_{0}+15 \ln \left(w+p_{0}\right)}\right]$

where $w_{0}=\left(E+M_{0} c^{2}\right) / m_{0} c^{2}$ and $\quad P_{0}=\sqrt{W_{0}^{2}-1 \text {. }}$

For the ground state positron group the available values of $W_{0}$ are 4. $70^{11,13}, 4.80^{12}$, all from beta-decay, and $4.75^{22}$ from $\mathrm{Zn}-68$ (pn) Ga-68 threshold. For the positron group to the $1.07 \mathrm{MeV}$ level the direct measure ment of Mukerji and Preiswerk ${ }^{11}$ gives $W_{0}=2.51$. However, the gamma ray energy together with the ground state $W_{0}$ fix this value uniquely. For the gamma ray measurements we have $1.02 \pm 0.02^{12}, 1.99 \pm 0.015^{23}, 1.088 \pm$ $0.005^{24}$, and our own value of $1.067 \pm 0.035$, all in $\mathrm{MeV}$. We adopt the val. ues. $W_{0}=2.59$ to the $1.07 \mathrm{MeV}$ level, and $W_{0}=4.70$ to the ground state. To compute theoretical $\mathrm{K} / \beta+$ ratio a numerical integration of the unscreened Fermi function has been carried out using NBS tables ${ }^{25}$. The screening correction for the positron was obtained from the curves of Perlman et al 9 . The screening correction for the $\mathrm{K}$ electron was taken into account by putting, $Z_{K}=Z_{\text {parent }}-0.3$. The computed $K / \beta+$ ratios are 1.42 (to the 1.07 $\mathrm{MeV}$ level) and 0.09 (to the ground state). After correcting for $8.5 \mathrm{o} / \mathrm{O} \mathrm{L}$ capture the Fierz interference terms are obtained as

$$
\mathrm{b}_{\mathrm{GT}}=+0.03 \pm 0.01 \text { and }-0.03 \pm 0.02
$$

to the ground state and first excited state respectively. The large uncertainty in the electron capture positron branching ratio for the ground state arises, apart from statistics, from the uncertainty in $N_{0}$ and the uncertainty in the value of $f_{\epsilon} 1 / f_{\beta}{ }^{1}$ itself. The more accurate value for the $1.07 \mathrm{Mev}$ level definitely shows the smallness of the Fierz term.

\section{g. Conclusions:}

Electron capture positron branching ratios have been measured fur the ground state beta-transition and the transition to the $1.07 \mathrm{MeV}$ level in $\mathrm{Zn}-68$, both of which are presumably pure Gamow-Teller transitions. On 
the basis of the ratio forthe $1.07 \mathrm{MeV}$ level, it is concluded that the Fierz term $b_{G T}=-0.03 \pm 0.02$. Although the measurement is not very accurate, the evidence for the smallness of the Fierz term is unmistakable. This conclusion is indeed consistent with the mor e accurate results on Co.58 and Na-22, also pure Gamow-Teller emitters, to be described in the following sections.

The measurements on Ga-68 have been presented briefly ${ }^{26}$ at the Cambridge Meeting of the American Physical Society and described in detail in Nuclear Physics 27.

\section{Electron Capture Positron Branching Ratio in the Decay of Co-58}

a. Introduction

72 day Co-58 decays by electron capture and positron emission to the $0.810 \mathrm{MeV}$ level in $\mathrm{Fe}-58$ followed by a gamma ray of this energy to the ground state. Besides; there is a weak electron-capture branch $(20 / 0)$ to the second excited state at $1.63 \mathrm{MeV}$. This level de-excites itself predominantly by the emission of a gamma ray of: $0.820 \mathrm{MeV}$ to the $0.810 \mathrm{MeV}$ level and partly by the emission of a gamma ray of $1.63 \mathrm{MeV}$ to the ground state of $\mathrm{Fe}_{-58}$. The decay scheme as given by-Frauenfelder et al. 28 is $x$ produced in Fig. 4. The end point of the positron spectrum is measured to be $0.472 \pm 0.006 \mathrm{MeV}^{29}$. No positron emission to the $\mathrm{O}^{+}$ground state of $\mathrm{Fe}$ 58 has been observed. The spin of $0.810 \mathrm{MeV}$ level is $2^{+}$from systematics of even-even nuclei ${ }^{14}$. The spin of the second excited state at $1.63 \mathrm{MeV}$ has been assigned $2^{+}$from angular correlation studies 28 . This is consistent with the presence of a cross-over gamma transition to the $0^{+}$ground state. The decay of Co-58 to the $2^{+}$states in Fe $\times 58$ and the absence of transition to the $0^{+}$ground state suggest a spin of $2^{+}$or $3^{+}$for Co-58. The spin has been directly measured to be 2 by pibrov and Jeffries ${ }^{30}$ by means of para. magnetic resonance experiments. The assignment of $2^{+}$to Co-58 makes the beta transition to the $0.810 \mathrm{MeV}$ level allowed by both Gamow - Teller and Fermi selection rules $(\Delta J=0$, No). However, recent nuclear orientation experiments of et al. ${ }^{31}$ have shown that the angular distribution of the $0.810 \mathrm{MeV}$ gamma ray is consistent only with the betta transition being pure Gamow-Teller, the amount of Fermi admixture being $0.003 \pm$ 
0.005 . Thus the measurement of electron capture to positron branching ratio to the $0.810 \mathrm{MeV}$ level becomes of obvious interest from the point of view of determining the Fiex z term.

Good et al: 32 and Cook and Tomnovbc ${ }^{33}$ have measured the ratio of total electron capture to positron emission in the decay of Co-58 to be 5.9 \pm 0.2 . When account is taken of the weak electron capture branching to the $1.63 \mathrm{MeV}$ level, the $\mathrm{K} / \beta+$ ratio to the $0.810 \mathrm{MeV}$ level becomes $5.8 \pm 0.2$. This result was obtained by comparison of the intensities of the annihilation radiation and the $0.81-\mathrm{MeV}$ gamma ray, and by a knowledge of the efficiencies. After the work to be described on Co-58 had been completed and briefly published ${ }^{34}$ by the author, the work of Konijn et al. ${ }^{35}$ on the same subject has come to attention. By using beta-gamma coincidence technique these workers determined the $\epsilon / \beta+$ ratio to be $5.67 \pm 0.14$.

Neglecting the weak electron-capture branch $(2 \mathrm{o} / \mathrm{o})$ to the 1.63 $\mathrm{MeV}$ level for the moment, the fraction of positrons in the decay of Co-58 can be expressed as $f_{+}=\beta / 2 c \sigma$, where $c$ is the singles counting rate for the 0.810 gamma ray, $\beta$ is the coincidence rate between the $0.810 \mathrm{MeV}$ gamma ray and the annihilation radiation, and $\sigma$ is the efficiency for detecting the annihilation radiation. The value of $f_{+}$when corrected for the presence of the weak branch will give the desired $\epsilon / \beta+$ ratio to the $0.810 \mathrm{MeV}$ level.

\section{b. Experimental}

Through the courtesy of Dr. R. W. Hayward of the National Bureau of Standards, a Co-58 source was made available for studies. Unfortunately this source contained an appreciable Co.60 impurity. Co-58 was evaporated onto a $0.0003^{\prime \prime}$ mylar foil and sealed.with celfophane. The sandwich was then squeezed between two lucite slabs each $1.3 \mathrm{~mm}$ thick and $1 \mathrm{~cm}$ square. The whole assembly was then sealed with black tape. Thus the positrons from Co-58 (0.470 MeV) were completely stopped. The $0.810 \mathrm{MeV}$ gamma ray was detected in a $1 \frac{1}{2} " \times 1 " \mathrm{NaI}(\mathrm{Tl})$ crystal. Source to detector distances of $1^{\prime \prime}$ to $1 \frac{1}{2}$ "were used. A typical singles gamma spectrum measured in the $2^{\prime \prime}$ cube crystal is shown in Fig. 5. Besides the annihilation radiation and the $0.810 \mathrm{MeV}$ gamma ray belonging to Co-58, gamma rays at 1.17 and 1. $33 \mathrm{MeV}$ are also prominently seen. The $1.63 \mathrm{MeV}$ gamma ray of Co-58 is too weak to be seen, and no effort was made to observe it. In order to determine the number of counts in the $0.810 \mathrm{MeV}$ photopeak, it is necessary 
to subtract the Cumpton background due to Co-60 gamma rays. In order to do this a pure Co 060 source was substituted and its spectrum was care fully normalized to that of Co-58,60. The dotted curve in Fig. 5 shows the normalized spectrum. For the coincidence measurements a single channel analyzer was set on the photopeak of the annihilation radiation and the spectrum in coincidence was obtained by gating the 20 -channel analyzer with the annihilation radiation. The coincidence spectrum thus obtained is shown in Fig. 6. It is observed that the coincident $0.810 \mathrm{MeV}$ gamma $r$ ay is superposed on a rather high background due to Co-60. In order to estimate and subtract this background, a coincidence spectrum was taken by re。 placing Co -58 by Co -60 and the spectrum normalized to the Co-58 spectrum. The resulting background was thus subtracted. In order to check on the re liability of this procedure, the $0.810 \mathrm{MeV}$ gamma ray was measured in triple coincidence with the two annihilation quanta. From this it was concluded that the background had been correctly estimated. The accidentals were about 10 percent of true coincidences in the doubles spectrum.

In order to determine $\sigma$, the efficiency for detecting annihilation radia. tion initially a calibrated $\mathrm{Na}^{22}$ source (accurate to $3 ; 0 / 0$ ) was used. By measuring the area under the photopeak and knowing the source strength one could compute the efficiency. A more accurate efficiency determination was made as follows: $\mathrm{A} \mathrm{N}^{13}$ source (a pure positron emitter of 10 minutes half life) was. produced by bombarding a 2 mil polyethelene foil for 10 minutes with 1 MeV deuterons at The Johns Hopkins University.Van de Graaff generator through the courtesy of O.N. Rask. After the bombardment the foil was cut into a tiny piece approximating the dimensions of the Co-58 source and sand wiched between two freshly cleaved NaI(Tl) crystals $1.2 \mathrm{~mm}$ thick and $1 \mathrm{~cm}$ square; and mounted in the same geometry as the Co.58 course. The beta spectrum observed in this system is shown in.Fig. 7. The energy calibration of the counter was made after the $\mathrm{N}^{13}$ source was dead by using external gamma ray sources of $\mathrm{Co}^{57}(0.123 \mathrm{MeV}), \mathrm{Cs}^{137}(0.661 \mathrm{MeV})$, and $\mathrm{Na}^{22}(1,28$ MeVl. A Fermi plot of the spectrum is shown in Fig. 8. It has an end-point of $1.16+0.05 \mathrm{MeV}$, in good agreement with the value of $1.20 \mathrm{MeV}$ in the literature $\mathrm{e}^{2 \mathrm{~s}}$. By following the decay of the activity for 3 half-lives, it was con. cluded that no impurities wexe present. Under the conditions of the bombaxd. ment, no other impurities were likely to be formed. 
The beta-spectrum was measured in coincidence with the annihilation

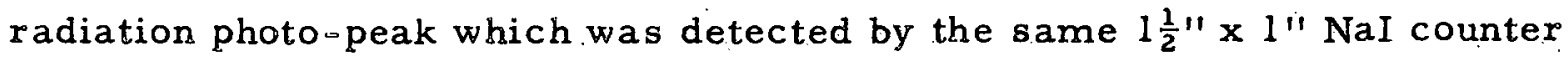
whose efficiency was to be determined. A portion of the beta spectrum is shown in Fig. 9. The efficiency for detecting the annihilation radiation is simply the ratio of the beta-spectrum in coincidence and in singles when corrected for decay. Further, since the crystal source was mounted on a light pipe a correction for the absorption of the $0.511 \mathrm{MeV}$ gamma-ray has. to be made. This is of the order of $3.7 \% / 0$. Since the positrons from Co$58\left(0.470 \mathrm{MeV}\right.$ ) and those from $\mathrm{N}^{13}$ (1.2 MeV) have different ranges in lucite and NaI respectively used to annihilate them, one might think that a correction for solid angle has to be made. However, the range of $0.470 \mathrm{MeV}$ positrons of $\mathrm{Co}^{58}$ in lucite is $1.4 \mathrm{~mm}$ and that of $1.2 \mathrm{MeV}$ positrons of $\mathrm{N}^{13}$ in $\mathrm{NaI}(\mathrm{Tl})$ is $1.16 \mathrm{~mm}$. The actual thickness used to annihilate the positrons were 1.3 and $1.2 \mathrm{~mm}$ respectively. A source to detector distance of $25 \mathrm{~mm}$ was used. In view of these circumstances the solid angle correction is less than $10 / 0$.

C. Results:

Table 2 lists the results obtained. The uncorrected $f_{+}$is $0.147 \pm$ 0.005. Referring to the Co-58 decay scheme (Fig. 4), it is seen that $1 \frac{1}{2}$ o/o of the $0.810 \mathrm{MeV}$ gamma rays arise from the $1.63 \mathrm{MeV}$ level, and another $1 \frac{1}{2} 0 / 0$ arise from cascading to the ground state. The uncorrected $f_{+}$has therefore to be multiplied by 0.03 to get the corrected value of $f_{+}$. In order to obtain the amount of electron-capture to the $0.810 \mathrm{MeV}$ level, it should be noticed that $2 \mathrm{o} / \mathrm{o}$ of the Co-58 transitions lead to the $1.63 \mathrm{MeV}$ level. Hence $E=0.98-0.151 \pm 0.005$. Thus the $\epsilon / \beta^{+}$ratio to the $0.810 \mathrm{MeV}$ level is computed to be $5.49 \pm 0.18$. The error introduced in the value of $20 / 0$ for the branching is very small.

\section{d. Discussion:}

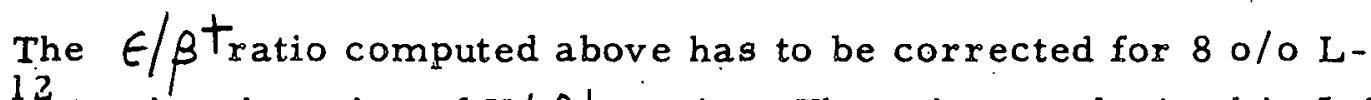
capture $\mathrm{e}^{12}$ to give the value of $\mathrm{K} / \beta+$ ratio. The value so obtained is 5.08 +0.17. The theoretical value is $5.15 \pm 0.24$ corresponding to maximum beta energy of $0.472 \pm 0.006 \mathrm{MeV}$. Thus our value is in excellent agree- 
Table 2. Summary of Results on $\mathrm{Co}^{58}$

(For symbols see text)

$$
\begin{aligned}
& c=91.00 \pm 1.12 \mathrm{cps} \\
& \beta=0.242 \pm 0.003 \\
& \sigma: \quad \text { (a) From Na}
\end{aligned}
$$

Source strength $=\mathrm{N}_{0}=$ no. of positrons $/ \mathrm{min}=(3.16 \pm 0.10) \times 10^{5} / \beta^{t} / \mathrm{min}$ $a=$ no. of cts. in the 0.5 .11 photo $\ldots$ peak $=47.0 \pm 0.0 \mathrm{cps}$ $\sigma=a / N_{0}=(8.92 \pm 0.33) \times 10^{-3}$

(b) From $\mathrm{N}^{13}$

$$
\begin{aligned}
& N_{\beta, 0.5}=358.0 \pm 6.0 \mathrm{cpm} \\
& N_{\beta}=23200 \pm 150 \mathrm{cpm} \\
& \sigma=\frac{1}{2} \frac{N_{\beta, 0.5}}{N_{\beta}}=(9.02 \pm 0.18) \times 10^{-3}
\end{aligned}
$$

$f_{+}($uncorrected $)=\beta / 2 c \sigma=0.147 \pm 0.005$

$f_{+}($corrected $)=(0.147 \pm 0.0 .05) 1.03=0.151 \pm 0.005$

$$
=0.980-f_{+}=0.829 \pm 0.005
$$

$$
\epsilon / \beta^{t}=\frac{0.829 \pm 0.005}{0.151 \pm 0.005}=5.49 \pm 0.18
$$

\footnotetext{
$\mathrm{L} / \mathrm{K}=0.08$

$\therefore K / \beta^{+}=5.08 \pm 0.17$
} 
ment both with theory and with previous measurements. As before, the Fierz term is computed from the expression

$$
b=\frac{R / R_{0}-1}{2\left[1+R / R_{0}\left\langle W^{-1}\right\rangle\right]}
$$

For Co-58 $\left\langle W_{-}^{-1}\right\rangle=0.76$ corresponding to $W_{0}=1.924$ $b=-0.006 \pm 0.014$

e. Conclusions:

The fraction of Co-58 decays by positron emission has been mea sured by coincidence methods using NaI crystals. The value is $0.151 \pm$ 0.005 . This value leads to a $\mathrm{K} / \beta+$ ratio of $5.08 \pm 0.17$ for the beta transition, to the $0.810 \mathrm{MeV}$ level. The theor etical $r$ atio is $5.15 \pm 0.24$. The Fierz term is computed to be $-0.004 \pm 0.014$. It is rather striking that the theoretical value of the $\mathrm{K} / \beta+$ ratio has a larger error than the measured value.

It follows then that the Fierz interference term is extremely small. Unfortunately $\mathrm{Co}-58$ is not the best case, since a small admixture of Fermi component in the beta transition may invalidate the conclusions reached so far. However, if it turns out, as is likely, that the Fermi component is zero, then it may be worthwhile to measure the end-point of the positron spectrum more accurately.

\section{A Reinvestigation of the Decay of $\mathrm{Na}-22$}

a. Introduction

2.60 Year. $\mathrm{Na}-22$ decays by positron emission and electron-capture to the first excited state of $\mathrm{Ne}-22$ at $1.28 \mathrm{MeV}$ followed by a gamma ray of this energy to the ground state. The decay scheme is shown in Fig. 10. The spin of $\mathrm{Na}-22$ has been measured to be $3^{36}$ and presumably the parity is even. The spirs of the $1.28 \mathrm{MeV}$ state of $\mathrm{Ne}-22$ is $2^{1}$ from life-time measurements ${ }^{37}$ and from systematics of even even nuclei ${ }^{14}$. Hence the transition $3^{+} \rightarrow 2^{+}$ follows the selection rule $\Delta \mathrm{J}=1$, No and is therefore pure Gamow-Teller. The electron capture to positzon branching ratio in the decay of $\mathrm{Na}-22$ has 
been extensively studied. A summary of previous work is given in a very recent paper by Konijn et al ${ }^{38}$. So far the best value reported is that of Sherr and Miller ${ }^{6}$ who obtained $\epsilon \beta^{\dagger}=0.110 \pm 0.005$ by an elegant experiment, and by comparing with the theoretical value of $0.1135 \pm 0.0020$ estimated the Fierz interference term to be $(-1 \pm 2)$ percent. Since all the present intexpretations on Fierz interference are based on this experiment we have been prompted to attempt a more precise determination of the ratio in $\mathrm{N} i 22$.

The principle of the present experiment is extremely simple. Suppose we have a gamma counter which detects the $1.28 \mathrm{MeV}$ gamma ray and a beta countêr which detects the positrons. Then assuming that all the positrons are counted, we can write for the beta -1.28 coincidences

and for the gamma ray

$$
\mathbf{N} \quad 1.28=A b \cdot f_{+} \sigma_{1.28}=a
$$

$$
\mathrm{N}_{1.28}=\mathrm{N}_{0} \sigma_{1.28}=\mathrm{b}
$$

where $\mathrm{N}_{0}$ is the transition rate

$$
f_{f}=\text { the fraction of decays by positron emission }
$$

and $\sigma_{1.28}=$ the efficiency for detecting $1.28 \mathrm{MeV}$ gamma ray. The ratio of a to be then yields $f_{+}$, from which the $\epsilon / \beta+$ ratio can be computed. This is possible provided the entire positron spectrum can be measured.

We have employed a $4 \pi$ plastic scintillation counter for detecting the positrons and a $\mathrm{NaI}(\mathrm{Tl})$ counter for the gamma $\mathrm{ray}$. The counter is biased to accept only the photopeak. The effectiveness of the $4 \pi$ scintillation counter for measuring the shapes of beta spectra has been demonstrated by the work of Johnson, Johnson and Langer ${ }^{39}$ and more recently by Robinson and Langer 40 and is substantiated by the present experiment.

A $\mathrm{Na}=22$ source from a $\mathrm{NCl}$ solution was evapqrated on a $0.0001 "$ mylar foil and covered with a similar foil. The $4 \pi$ counter was formed in. the following way. Two plastic cylinders each $3 \mathrm{~mm}$ thick and $1 \mathrm{~cm}$ in diameter were chosen. One of the cylindres had a depression $\frac{1}{2} \mathrm{~mm}$ deep and $\frac{1}{2} \mathrm{~cm}$ in diameter. The $\mathrm{Na}-22$ sandwich was placed in the depression. The two cylinders were pressed together to form the $4 \pi$ counter. A cone-shaped light pipe $1 \frac{1}{2} "$ long having a well at the apex was mounted on a DuMont 6292 phototube. To the bottom of the well the $4 \pi$ plastic scintillation crystal was 
cemented by means of Canada balsam. The sides of the well had been painted white to ensure good light collection. The top of the well had a thin aluminum foil whitened inside. The gamma counter was a 2 "' cube $\mathrm{NaI}(\mathrm{Tl})$ crystal which had a resolution of $11 \mathrm{o} / \mathrm{o}$ for $0.661 \mathrm{Mev}$ gamma ray of $\mathrm{Cs}-137$. The $4 \pi$ counter had a resolution of $16.0 / 0$ for the $0.624 \mathrm{MeV}$ conversion line of Cs-137. The entire assembly of crystal and counters was surrounded by 2 " of lead at a distance of 4 ".

\section{b. Exper imental:}

The general features of the $4 \pi \quad \beta$ counter were investigated by a $\mathrm{P}^{32}$ source using plastic cylinders each $5 \mathrm{~mm}$ thick and $1 \mathrm{~cm}$ diameter.A Fermi plot of the spectrum is shown in.Fig. 11. The end-point of $1.72 \mathrm{MeV}$ is in good agreement with the literature ${ }^{29}$. Experiments on $\mathrm{Na}^{22}$ were started with plastics of the dimensions described above. The gamma counter was set on the photo-peak of the $1.28 \mathrm{MeV}$ gamma ray. The peak had a width of 3.5 volts at 35 volts. This was used to gate the 20 -channel analyzer. The positron spectrum coincident with the $1.28 \mathrm{MeV}$ gamma $\mathrm{ray}$ is shown in Fig. 12. Energy calibration of the spectrometer was obtained by using external gamma rays of $\mathrm{Na}-22(0.51 \mathrm{l} \mathrm{MeV})$ and $\mathrm{Cs}-137(0.661 \mathrm{MeV})$. The Compton edges 10$c$ ated at $3 / 4$ of the maximum were used. The calibration is also shown in Fig. 12. The calibration curve intercepted the axis corresponding to zero pulse height at $18 \mathrm{Kev}$ in agreement with similar observations by Johnson, Johns on and Langer 39 .

Because of the fact that the plastic chosen had dimensions somewhat greater than the range of positrons, one wo uld expect that the observed beta spectrum may not be the correct one, but somewhat distorted by the simultaneous detection of a beta particle and its associated compton. Thus the ef fect would be qualitatively to shift the spectrum towards high energy, without changing the area under the spectrum.

In order, therefore, to obtain the undistorted spectrum, the beta spectrum was measured in triple coincidence with the $1.28 \mathrm{MeV}$ gamma ray and the two annihilation quanta. The experimental arrangement and a functional diagram of the electronic circuitry are shown in Fig. 13. Pulses from the two $0.511 \mathrm{MeV}$ counters and the $1.28 \mathrm{MeV}$. counter were fed to a triple coincidence circuit. The output from the triple coincidence circuit was used 
to gate the 20-channel analyzer. The positxon spectrum gated by the triples is shown in Fig. 12, normalied to the doubles spectrum beyond $50 \mathrm{keV}$. The statistical error for each point varied from 2 to $40 / 0$. The spectrum is indeed displaced, as expected. To obtain a quantitative justification for the spectral displacement, the positrons were completely stopped in just enough. lucite and the Compton distribution was obtained in coincidence with the anni hilation radiation and the $1.28 \mathrm{MeV}$ gamma ray. The spectrum thus obtained is shown in Fig. 12 and is similar to the one that is obtained using an external gamma source except for the absence of edge effects.

If the assertion that the effect of the Compton distribution due to annihilation radiation is simply to shift the doubles spectrum is correct; then it must be possible to express the doubles spectrum $d(h)$ in terms of the triples $t(h)$, and the Compton distribution $C\left(h-h^{\prime}\right)$. That.is, we should be able to write

$$
d(h)=\sum_{h^{\prime}=0}^{h} t\left(h^{p}\right) C\left(h-h^{\prime}\right) \Delta h .
$$

A numerical calculation was carried out to test this assumption. For an as sumed compton of $6 \mathrm{o} / 0$ the agreement from point to point $3 \times 4 \mathrm{o} / \mathrm{o}$. The as sumption of $6 \mathrm{o} / 0$ compton is not inconsistent with the dimensions of the plastic and the compton cross section. (The choice of $60 / 0$ is not critical, since the triples spectrum itself was known to $2.4 \% / 0$. The agreement thus obtained provides quantitative justification for the as sertion made earlier. It must be pointed out in this connection that the effect of inner bremsstrahlung is to displace the spectrum in a direction opposite to that of the comp. ton distribution, but because of the weakness of the effect the Compton effect predominates. The preservation of areas in the doubles and triples spectrum is indicated by the fact that the two areas could be normalized to within $1 / 10$ of a percent.

Since the lowest energy observed was around $40 \mathrm{KeV}$, an extrapola: tion to zero energy has to be made in order to obtain the area under the whole spectrum. To do this the following procedure was adopted: The ideal Fermi spectrum corrected for screening was plotted. The spectrum was distorted for finite resolution. (16 \%/o in our case) at various points of the spectrum by folding in a gaussian of the proper width. The assumption.was made that the half width varied as the square root of the energy over the entire energy range. Choosing various energies (h $\mathrm{min}$ ), the area to the right of $h_{\text {min }}$ was 
obtained. It was determined that below $50 \mathrm{KeV}$ the area under the beta spec. trum with and without resolution correction differed only by $1 / 10$ percent and amounted to 5.3 o/ of the area under the $\beta$ spectrum beyond $50 \mathrm{KeV}$. Thus the area under the ideal Fermi distribution was taken as the correct area. This when added to the area due to the remaining portion of the doubles spectrum (which had been corrected by the compton to get the undisplaced spectrum) would give the total area.

In order to test for any possible systematic exrors, the ratio of area to the right of $h_{\text {min }}$ and the entire area from $50 \mathrm{KeV}$ up to the maximum en. ergy was plotted both for the ideal Fermi spectrum corrected for finite resolution, and the actual observed doubles spectrum corrected to the triples spec. trum, as a function of $h_{\text {min }}$. The result is displayed in.Fig. 14. It is seen that the data of four different $r$ uns are consistent within themselves to $1 \frac{1}{2} 0 / 0$ and consistent with theoretical plot within $1 \% / 0$. This gives confidence that there are no systematic errors involved.

The experiments were repeated with and without lead shielding. The elffect. of channel width on the gamma ray side was next studied. A differ ent source source was made and the experiment repeated. In each case consist ent results were obtained. Throughout the course of the experiment the count: ers were periodically checked. The energy calibration of the beta counter was carried out before and after each run. The overall statistics in each run was $1 / 10$ to $2 / 10$ of a percent. Altogether seven $r$ uns were made.

c. Results:

The data from the six runs are assembled in Table 3 together with explanation. The total area in the beta spectrum up to $50 \mathrm{KeV}$ is $164.08 \pm$ $0.18)$ cps. This when corrected for the remaining portion of the spectrum becomes $(67.52 \pm 0.19) \mathrm{cps}$. From this area and the area under the 1.28 $\mathrm{MeV}$ gamma say $(75.10 \pm 0.11) \mathrm{cps}$, the value of $f_{+}$, the fraction of $\mathrm{Na}-22$ decays by positron emission, is computed to be $0.899 \pm 0.003$. This yields an average ratio of

$$
\epsilon / \beta^{+}=\frac{1-(0.899 \pm 0.003)}{(0.899 \pm 0.003)}=0.112 \pm 0.004 .
$$

Apart from statistical error; the other uncertainty is due to the folding of Compton distribution, and in the estimation of the area under the 
beta and gamma spectrum. A calculation was made to see how much error would be introduced if the half width of the gaussian curve deviated from obeying $\sqrt{E}$ law. Dependences proportional to $E^{0.4}$ and $E^{0.6}$ were tried. From this it was concluded that the error introduced is less than $1 / 10$ of a percent in the final result.

An error in the determination of the end-point of the positron spec. $t$ rum would introduce an error in the value of $h_{\text {min. }}$ Because of the assumed linearity in energy scale this would tend to introduce a linear systematic er ror. In Table II the end-points are tabulated with uncertainties.

Table II

$\begin{array}{cc}\text { Run No. } & \text { End - Point (KeV) } \\ 1 & 546 \pm 11 \\ 2 & 541 \pm 10 \\ 3 & 548 \pm 11 \\ 4 & 539 \pm 10 \\ 5 & 540 \pm 10 \\ 6 & 544 \pm 11\end{array}$

From the above table the systematic error introduced in this way is estim. ated to be less than 1.2 o/o. Thus allowing for this error, our ratio would at worst become

resulting in the Fierz term

$$
\epsilon / \beta^{+}=0.112 \pm 0.005
$$

$$
{ }^{b T}=-0.004 \pm 0.013 \text {. }
$$

\section{d. Discussion:}

The computed value of $\epsilon / \beta+$ is somewhat better than that of Sherr and Miller ${ }^{6}$. The theoretical value of $\epsilon / \beta^{t}$ is $0.1135 \pm 0.0020$ when cor rected for screening and $65 \% / 0 \mathrm{~L}$-capture 10 . The value of $\left\langle\mathrm{w}^{-1}\right\rangle_{\text {for }} \mathrm{Na}$. 22 for $W_{0}=2.061$ is 0.7 . The Fierz term is computed as before from the expression 
Table 3: $\mathrm{Na}^{22}$ Data

$\begin{array}{llll}\text { (a) (b) } & \text { (c) }\end{array}$

$\begin{array}{lccc}\text { Run } & \text { Conditions } & \beta_{1} 1.28>50 \mathrm{KeV} & 1.28>0 \mathrm{KeV} \\ \text { No. } & \text { cps } & =1.053(\mathrm{a}) \mathrm{cps}\end{array} \quad \mathrm{N}_{1.28} \mathrm{cps} \quad f_{+}=(\mathrm{b}) /(\mathrm{c})$

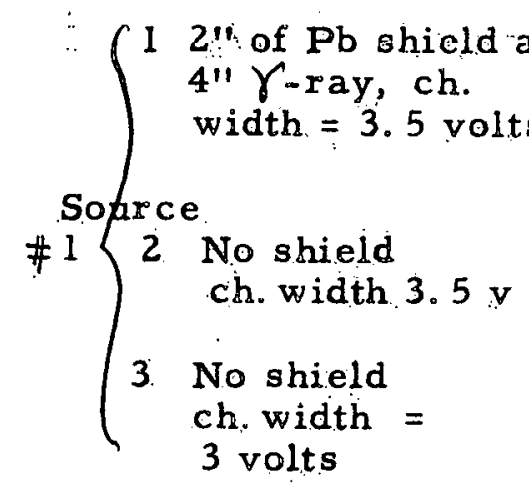

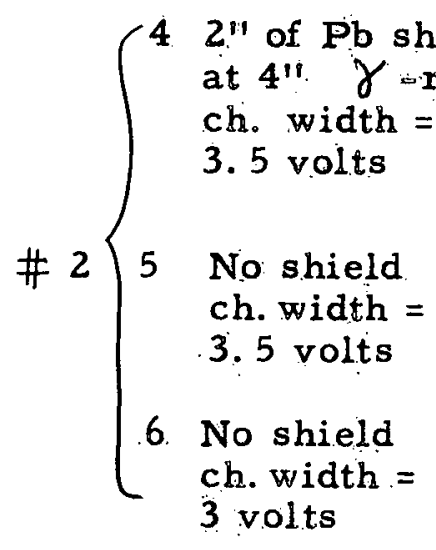

$57.82 \pm 0.22 \quad 60.88 \pm 0.23 \quad 67.76 \pm 0.15 \quad 0.899+0.004$

$64.23 \pm 0.19 .67 .63+0.20 \quad 75.24+0.15 \quad 0.900+0.003$

$64.08+0.1867 .48 \pm 0.19 \quad 75.21 \pm 0.11 .0 .898 \pm 0.003$

$48.31 \pm 0.14 \quad 50.87 \pm 0.15 \quad 56.02 \pm 0.10 \quad 0.900+0.003$

$48.07 \pm 0.17 .50 .62 \pm 0.18 \quad 56.3 l^{\prime+1.11} \quad 0.899+0.004$

$42.78+0.13 \quad 45.05+0.14 \quad 50.11 \pm 0.10 .0 .899 \pm 0.003$

Average value of $\mathrm{f}_{+}=$

$0.899 \pm 0.003$

$$
\begin{aligned}
& \epsilon=1-f_{+}=0.101 \pm 0.003 \\
& \therefore \epsilon / \beta+=\frac{0.101 \pm 0.003}{0.899 \pm 0.003}=0.112 \pm 0.004
\end{aligned}
$$




$$
\mathrm{b}_{\mathrm{GT}}=\frac{R / R_{0}-1}{2\left[1+\mathrm{R} / \mathrm{R}_{0}\left\langle\mathrm{~W}^{-1}\right\rangle\right]}=\frac{\frac{0.112 \pm 0.004}{0.1135 \pm 0.0020}-1}{2\left[1+\frac{0.112 \pm 0.004}{0.1135 \pm 0.0020}\right]}{ }^{1}(0.7)
$$

The result is

$$
{ }_{G T}^{b}=0.004 \pm 0.012
$$

$\mathrm{Na}-22$ is perhaps the ideal case for determining the Fierz term because of the low $Z$ involved. It is very unfortunate that the end point of the beta spec. trum is not known. well enough to attempt any further. refinement in experimental techniques to measure $\epsilon / \beta^{\dagger}$ ratio. In any case it has been deminstrated. that the plastic scintillator can be effectively used in the study of beta spectra and precision results obtained if analyzed with caution.

\section{e. Conclusions:}

A reinvestigation of the electron capture to positron branching ratio in the decay of $\mathrm{Na}-22$ has been made with greater. precision than has been pos sible before, using a $4 \pi$ plastic scintillator and a gamma counter. The measured value for $\epsilon / \beta+$ is $0.119 \pm 0.004$. From this the Fierz interference term is estimated to be ${ }^{b}{ }_{G T}=-0.004 \pm 0.012$. It is suggest ed that the beta spectrum end-point be measured with greater precision to make much moxe meaningful estimates of the Fierz term. It would be of interest to measure

$\mathrm{K} / \beta+$ ratios in unique forbidden transitions allowed only by Gamow-Teller selection rules.

\section{Conclusions to Part A.}

K-capture to positron branching ratios have been measured in three pure Gamow-Teller transitions (allowed), namely Ga-68, Co-58, and Na-22 using coincidence scintillation spectrometer techniques, special attention being given to the study of $\mathrm{Na} 22$. The measured ratios are $1.28 \pm 0.12$ (For $\mathrm{Ga}-68$ ), $5.08 \pm 0.17$ (for $\mathrm{Co}-58$ ) and $0.105 \pm 0.004$ (for Na-22). By compar ing these ratios with the theoretical values the Fierz interference term can be computed. The results for $b_{G T}$ are $-0.03 \pm 0.02$ from Ga $-68,-0.004$ \pm 0.14 from $\mathrm{Co}-58$, and $-0.004 \pm 0.012$ from $\mathrm{Na}-22$. Considering the more 
precise measurements on $\mathrm{Co}-58$ and $\mathrm{Na}-22$, one is led to the conclusion that the Fierz interference term in allowed Gamow-Teller transitions is extremely small, practically zero. The rather less precise results for Ga-68 are consistent with this interpretation. It must be pointed out that our result for $\mathrm{K} / \beta+$ ratio in the decay of $\mathrm{Na}-22$ is slightly better than the value of Sherr and Miller, who had obtained the best result so far. Our measurement can be considered as representing a limit on the precision attai nable with plastic scintillators. A further refinement in technique can be consider ed worthwhile only when refinements in the determination of the spectral end-points are made -. when the end-point of $\mathrm{Na}-22$, for example, can be measured to $1 / 10$ percent.

5. Summary of Fierz Interference in Beta-Decay.

In this section the conclusions regarding Fierz interference in GamowTeller transitions as indicated by our measurements are summarized (see Table 4).

Table 4. Summary of Results on Fierz. Term from This. Work

\begin{tabular}{llllc} 
Nucleus & \multicolumn{2}{l}{ Transition } & $\mathrm{W}_{\mathrm{O}}$ & $\mathrm{b}_{\mathrm{GT}}$. \\
$\mathrm{Ga}-68$ & $1^{+}$ & $2^{+}$ & 4.70 & $-0.03 \pm 0.02$ \\
$\mathrm{Co}-58$ & $2^{+}$ & $2^{+}$ & 1.924 & $-0.004 \pm 0.014$ \\
$\mathrm{Na}-22$ & $3^{+}$ & $2^{+}$ & 2.061 & $-0.004 \pm 0.012$
\end{tabular}

Gerhart ${ }^{3}$ has made an excellent analysis of Fierz interference in Fermi transitions and concludes $b_{F}=0.00 \pm 0.12$. A brief review of Fierz interference in beta-decay has been recently given by the author ${ }^{41}$. From the above table one sees that the best evidence for the smallness of the Fier $z$ term in G-T interactions comes from Na-22. Konijn et al. ${ }^{38}$ have summarized data regarding the interference term as determined by $\mathrm{K} / \beta+$ ratio tecpnique. They conclude that $b_{G T}=-0.007 \pm 0.010$.

From evidence presented above for Gamow-Teller transitions and from Gerhart's analysis of Fermi transitions one can conclude that the Fierz term is practically zero. Before parity non-conservation was discovered, the Fierz term in $G-T$ transitions could be expressed as 


$$
{ }^{B} G T=\frac{C_{A} C_{T}}{\left|C_{A}\right|^{2}+\left|C_{T}\right|^{2}}
$$

The smallness of $\mathrm{b}$ could be interpreted as implying that either $\mathrm{C}_{\mathrm{A}} / \mathrm{C}_{\mathrm{T}}$ or $\mathrm{C}_{\mathbf{T}} / \mathrm{C}_{\mathrm{A}}$ was small. With the discovery that parity is not conserved in betadecay, the definition of $b$ has acquired the extended form

$$
{ }_{G T}^{b}=\frac{\operatorname{Re}\left(C_{A} C_{T}+C_{A}{ }{ }^{*} T_{T}{ }^{\prime}\right.}{\left|C_{A}\right|^{2}+\left|C_{T}\right|^{2}+\left|C^{i}\right|^{2}+\left|C^{i}\right|^{2}}
$$

where $C_{A, T}$ are the parity conserving coupling constants

$C_{A^{\prime}, T^{\prime}}$ are parity violating coupling constants, and the *s denote com plex conjugation resulting from a possible violation of time reversal invariance.

With this new definition the smallness of only means

$$
\operatorname{Re}\left(C_{A} C_{T}^{*}+C_{A^{\prime}} C_{T}{ }^{* *}\right)=0
$$

This implies that

$$
\frac{\mathrm{C}_{\mathrm{A}}}{\mathrm{C}_{\mathrm{A}^{\prime}}}=-\frac{\mathrm{C}^{\mathrm{i}} \mathrm{T}^{*}}{\mathrm{C}^{*} \mathrm{~T}}
$$

Nothing more can be said concerning the coupling constants unless the relation between the parity conserving and parity non-conserving coupling constants is known, and further a knowledge about the validity of time reversal invariance. The loss of definitiveness regarding the significance of the Fier $z$ term is one of the major consequences of the discovery of parity non-conservation. 


\section{PART B}

\section{Search for A Low-Lying $0^{+}$State in Ga-68}

\section{A. Introduction}

The decay of 270 day Ge-68 has been studied by Crasemann 12 and by Hor en ${ }^{18}$. The spin of Ga-66 has been measured to be $0^{42}$ and presumably the parity is even. The spin of Ga-68 is most likely to be $1^{+}$as indicated by allowed $\log \mathrm{ft}$ values for positron decays to $\mathrm{Zn}-68$. This assignment. has been confirmed by a recent direct spin measurement by Hubbs et al 17 . In this region of nucleon numbers $31-37$ the $p_{3 / 2}$ and $\$_{5 / 2}$ are close-lying in energy, as is evident from the measured spins of some stable isotopes in. this region: ${ }^{43}$. Although the decay energy for Ge-6.8-Ga-68 is nearly $700 \mathrm{KeV}^{20}$, no gamma rays have been reported in the decay nor any internal conver sion electrons. Levels in Ga-68 at $0.17,0.34$, are known through the reaction $\mathrm{Zn}-68(\mathrm{p}, \mathrm{n}) \mathrm{Ga}-68^{22}$. The absence of gamma rays in the decay of Ge-68 means that none of the se levels are populated, which in turn implies that the spins of these states must $\geqq 2$ if of even parity. On the other hand, the closeness of $\mathrm{p}_{3 / 2}$ and $\mathrm{f}_{5 / 2}$ suggests that there might exist a low-lying $0^{+}$state formed. by the same configuration as is found in Ga-66. Such a competition between $p_{3 / 2}$ and $f_{5 / 2}$ is in evidence in $\mathrm{Cu}-62$, which has a low-lying $0^{+}$level very close to the $1^{+}$ground state ${ }^{44}$. Since no measurements on the low-energy radiations had been reported, it seemefof interest to look for a low-lying $0^{+}$ state. If indeed such a level did exist, it was expected that the electron capture decay from Ge-68 to both $0^{+}$and $1^{+}$states would be allowed and would result in a gamma-ray (MI) which could be detected by X-ray-X-Ray and/or $X-r a y-g a m m a$ ray coincidences, if not in the singles gamma spectrum.

An odd-odd nucleus can be looked upon as made up of a superposi.tion of two odd $A$ nuclei, one of them with an odd number of neutrons and the other with an odd number of protons. Since the angular momentum according to the shell model seems to be carried entirely by the last odd nucleon, the study of the spins of odd-odd nuclei throws light on the nature of the nuclear force between the odd proton and the odd neutron. By assuming various forms for the neutron-proton interaction the energy levels of an odd-odd nucleus can be theoretically predicted. The experimental verification of such theoretical predictions is rather hard, since odd odd nuclei as a rule are unstable. Therefore it would be of interest to study the energy 
fievels of as many odd-odd nuclei as possible. From this point of view the search for a low-lying $0^{+}$state in Ga-68 seemed more meaningful.

\section{B. Experimental}

A Ge -68 source in equilibrium with Ga -68 was produced as described under Ga-68 in Part A of this report. The region (0 to $30 \mathrm{KeV}$ ) was studied using very thin $(\sim 1 \mathrm{~mm}$ thick and $1 \mathrm{~cm}$ square) NaI(Tl) crystals. The crys tal was mounted with silicone on a DuMont 6292 phototube and sealed with an 0.0001 " aluminum foil. The foil bepides ensuring a high transmission for the soft radiations, also served as light reflector. Fig. 15 (curve a) shows a typical spectrum. The resolution for $9 \mathrm{KeV} \mathrm{K-X-ray} \mathrm{varied} \mathrm{from} 6.0$ to 60 o/o; depending on the crystal. The $5.8 \mathrm{KeV} \mathrm{K-X-ray} \mathrm{of} \mathrm{Fe-55} \mathrm{served} \mathrm{as} \mathrm{energy}$ standard. Clearly there is no indication of any gamma ray. It is further ap.. parent that the contribution of high energy pulses to the $\mathrm{X}$-ray is appreciable. The region $30 \mathrm{KeV}$ to $1.2 \mathrm{MeV}$ was investigated by a $1 \frac{1}{2} "$ x 1 ". NaI(Tl) crystal. Besides the annihilation radiation and the known gamma ray whose energy was measured to be $1.067 \pm 0.035 \mathrm{MeV}$, no other gamma ray was observed $(<8 \mathrm{o} / 0$ of $1.07 \mathrm{MeV}$ gamma ray). To study $\mathrm{X}$-ray $\mathrm{X}$-ray coincidences the Ge-68 source was sandwiched between two DuMont 6292 phototubes on which thin NaI(TI) crystals were mounted, covered with aluminum foil, and sealed with a light shield. Fig. 15 (curve c) shows the coincidence spectrum $(0$ to $30 \mathrm{KeV})$. Most of the counts above accidentals are due to crystal to crystal scattering of higher energy pulses. This was confirmed by attenuating the $\mathrm{K}-\mathrm{X}$-ray in one of the channels and repeating the spectrum. The $1.07 \mathrm{MeV}$ gamma ray is too feebly converted to give any noticeable coincidences. The $\mathbf{X}-\mathbf{r}$ ay high energy gamma ray (up to $750 \mathrm{KeV}$ ) coincidences were performed by replacing one of the counters by a $1 \frac{1}{2} " x 1 ! \mathrm{NaI}(\mathrm{T})$ ) counter. The results were negative. The region beyond $750 \mathrm{KeV}$ was not studied, since the Ge-68 - Ga-68 decay energy can at most be $750 \mathrm{KeV}^{22}$.

A more rigorous search for the $0^{+}$state was attempted by separating Ge from Ga chemically, using successive ether extractions from $6 \mathrm{~N} \mathrm{HCl}$. Since an appreciable part of Ge $(40-60.0 / 0)$ is also extracted ${ }^{45}$ into the ether layer, and since the number of extractions could not be done more than twice owing to the fast growth of 68 minute Ge-68, an efficiency of $50 \mathrm{o} / 0$ or less was all that could be attained for Ge. The Ge thus separated was converted 
into the sulphide and rapidly transferced to the counting system. The region $0.30 \mathrm{KeV}$ was studied as a function of time with the 20-channel analyzer using a thin freshly cleaved NaI crystal. At the same time a second counter, $1 \frac{1}{2}$ " $\mathbf{x} \cdot 1 "$ ( $\mathrm{NaI}(\mathrm{Tl})$ was set on the photopeak corresponding to the annihilation radiation and its growth in Ga-68 was followed as a function of time. Fig. 15 (curve b) shows the pulse height spectrum taken immediately after the chemical separation. Clearly there is no gamma ray $(<10 / 0$ of $\mathrm{K}-\mathrm{X}-\mathrm{ray})$. From the growth curve of annihilation radiation which corresponded to a 70 minute half life, an upper limit of 0.4 o/o per decay could be set on positron emis sion by Ge-68. Horen 18 arrived at an upper limit of 0.9 o/o by comparing the gamma spectrum of Ge-68+Ga-68 with that of Ga-68 alone.

\section{Discussion:}

The failure to observe any $\mathrm{K}$ X-ray $\mathrm{K}-\mathrm{X}$-ray, low-energy gamma ray or $\mathrm{K}-\mathrm{X}$-ray high energy gamma ray (up to $750 \mathrm{KeV}$ ) coincidences suggests that the $0^{+}$state lies either very close to the ground state at an energy less than the $\mathrm{K}$-binding energy, or very high - - higher than the Ge-Ga decay energy. The absence of transitions to any of the known excited states in Ga-68 confirms that the spins of these states must be $\geqslant 2$ if of even parity.

\section{Conclusions:}

A search for a possible low-lying $0^{+}$state in $\mathrm{Ga}-68$ has been made through the decay of Ge 68 using NaI(Tl) crystals. The results were negative. The failute to find the $0^{+}$state implies that the analogy of $\mathrm{Cu}-62$ does not hold in this case. The absence of positrons in the decay of Ge-68 is consistent with the decay proceeding purely by electron-capture.

Results of this investigation have been published in Nuclear Physics 27.

\section{Search for EO Transiftion from the $2.3 \mathrm{MeV}$ Level in $\mathrm{Zn}-68$}

a. Introduction

The decay of Ga 68 has been. recently reinvestigated by Horen. ${ }^{18}$, who found evidence for levels in $\mathrm{Zn}-68$ at $1.07,1.88$ and $2.3 \mathrm{Mev}$. The decay scheme as. given by Hoxen is reproduced in Fig. 16. The branching percent- 
ages to the various levels are indicated, together with the gamma-ray branchings. The levels were assigned the following spins and paritics re: spectively: $\left(1.07\left(2^{+}\right), 1.88\left(1^{+}\right.\right.$or $\left.2^{+}\right)$, and $2.3\left(2^{+}\right)$. No cross-over gamma ray transition from the $2.3 \mathrm{MeV}$ level to the $0^{+}$ground state was observed. This fact together with the result of angular correlation studies on the 1.071. $24 \mathrm{MeV}$ gamma cascade led Horen to suggest $2^{+}$for the $2.3 \mathrm{Mev}$ level. Since the errors on the angular correlation data were rather large, this spin assignment was considered to be far from unambiguous. It seemed attractive to assign $0^{+}$for this state. Such an assignment would indeed be consistent with all the observed data. Since $0-0$, No transitions throw light on the nuclear structure involved, it seemed worthwhile to look for additional evidence in support of this hypothesis.

$0-0$, No gamma transitions are strictly forbidden because of the transyerse nature of the photon. Hence the de-excitation of the state in ques.$t$ ion can proceed only through internal conversion. If, however, the energy of the transition exceeds $2 \mathrm{~m}_{\mathrm{o}} \mathrm{c}^{2}$, an alternative mode of decay is possible. namely internal pair formation. The transition probability for an Eo transition can be expressed as a product of electronic and nuclear wave functions as in the case of internal conyersion for higher multipoles. Thomas ${ }^{46}$ has given expressions for the processes of $\mathrm{K}$-conversion and internal pair-creation. The nuclear matrix element is expressed as

$$
M=\int \Psi_{\text {in }}^{*} \sum_{p} n_{p}^{2} \Psi_{\text {fin }}
$$

where the summation extends over all the protons in the nucleus. M can be conveniently written as $M=\int R^{2}$ where $R$ is the nuclear radius and $\rho$ is a measure of the strength of the EO transition. a quantity analogous to the reciprocal of the $f t$ value in beta mecay. The theoretical transition rates. for $\mathrm{K}$-conversion and pair formation according to Thomas ${ }^{46}$ are given by

$$
W_{k} / \rho^{2}=7.6 \times 10^{14} \frac{[S+\sqrt{3}(S+2)]^{2}}{[\Gamma(3+2 S)]^{3}}(\delta, z)^{3+2 S} 2 R^{4+4 S} \frac{\varepsilon+1+S}{\eta} f(\eta, z)
$$

and

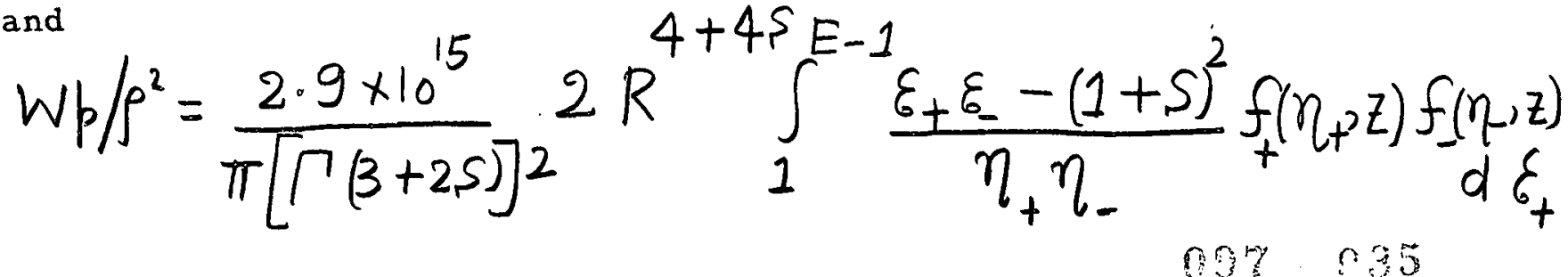


where $\varepsilon_{+}+\varepsilon=E ; \quad \varepsilon=E+1+S ; \eta=\left(\varepsilon^{2}-1\right)^{1 / 2} ; \quad S=\sqrt{1-\alpha^{2} z^{2}}-1$ $f_{+}$is the Fermi function as tabulated in the NBS tables ${ }^{19}, E$ is the transition energy. All the units are relativistic. $R=1.3 \times 10^{-13} \mathrm{~A} / 3 \mathrm{~cm}$. Church and Weneser 47 have given graphs of $W_{K}$ as a function of the atomic number, $Z$, and transition energy $E$.

$0^{+}$first excited states are known in the doubly magic nuclei $-{ }_{8} \mathrm{O}^{16}$, ${ }_{20} \mathrm{Ca}^{40}$, and $40^{\mathrm{Zr}^{90}}$ with the neutrons in $\mathrm{Zr}^{90}$ completely filling the $\mathrm{p}_{1 / 2}$ shell. $0^{+}$second excited states occur in ${ }_{6} \mathrm{C}^{12}, 32^{\mathrm{Ge}} \mathrm{e}^{72}$ and $46^{\mathrm{Pd}} 106$ 0.0

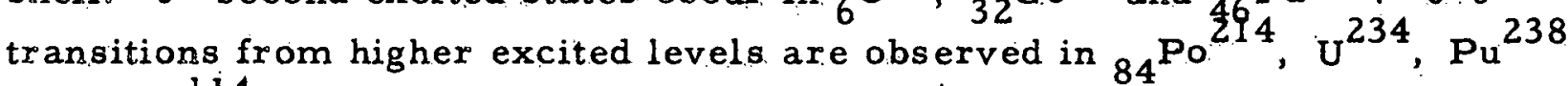
and ${ }_{48} \mathrm{Cd}^{114}$. It has been suggested that the $0^{+}$excited state in doubly magic nuclei may be due to two particle excitation. A similar explanation has been advanced for the second excited state of $\mathrm{C}^{12}$.

\section{b. Experimental}

Since the internal pair positron spectrum from the $2.3 \mathrm{MeV}$ level in $\mathrm{Zn}-68$ would be superposed on a rather high background of positrons from the beta-decay of Ga-68, it was decided to look for internal conversion electrons of 2. $3 \mathrm{MeV}$ which would appear as a monoenergetic line well beyond the positron end point of $1.88 \mathrm{MeV}$. In order to observe the small queak expected due to the conversion electrons, it was necessary to reduce the background to a minimum. In the present investigation this has been accomplished by the use of an anti-coincidence arrangement.

A block diagram of the experimental arrangement is shown in Fig. 17. The Ga 48 source was. sandwiched between two plastic scintillators each $1 \frac{1}{2} \mathrm{~cm}$ high and $3 \mathrm{~cm}$ in diameter (thus ensuring $4 \pi$ geometry) and mounted on: the face of a DuMont 6292 photomultiplier tube. A second plastic scintillator surrounding the first one was mounted on another phototube ans served as the anti-coincidence counter. The sensitive volume of the counters was surrounded by 4 inches of lead. Pulses in counter 1 not accompanied by pulses in counter 2. operated a gate which admitted to the 20 channel analyzer any pulse in the beta counter (counter 2 ) occurring in an interval of 3.5 microseconds. The anti-coincidence circuit used here was the same as employed by Tilley 48 
with some slight modification. The modification consisted in reducing the duration of the univibrator pulse from 12 to 3 microseconds.

c. Results:

The positron spectrum observed in this system is shown in Fig. 18. The end-point of the spectrum was determined to be $1 / 89 \pm 0.06 \mathrm{MeV}$ by comparis on with the beta-spectrom of P-32 of end-point 1. I I MeV, also shown in the figure. The resolution of the counter was $18 \% / 0$ for the $\mathrm{Cs}-137624 \mathrm{KeV}$ conversion line. The search for internal conversion electrons was made by setting the pulse height at 2. $1 \mathrm{MeV}$. The search lasted 105 hours, during which time the apparatus was periodically checked. From the total number of counts observed in the region where the conversion electrons were expected with and wi thout the source, and the total number of counts in the beta spectrum, an upper limit of $(5 \pm 25) \times 10^{-9}$ conversion electrons per decay of Ga 68 (assuming that the positrons account for $88 \mathrm{o} / 0$ of the transitions) could be set. The results are summarized in Table 5 .

\section{d. Discussion and Conclusions}

One can calculate the expected yield of 2. $3 \mathrm{MeV}$ internal conversion electrons as follows (assuming that the state is $0^{+}$). On the basis of the single particle model, the transition probability for an electric quadrupole (E2) gamma transition is given by Moszkowski ${ }^{49}$ as $W \gamma=1.6 \times 10^{8} \mathrm{~A}^{1 / 3} \mathrm{E}_{\gamma}^{5}$. For the 1.24 MeV gamma ray from the 2. $3 \mathrm{MeV}$ level to the $1.07 \mathrm{MeV}$ level this is calcul ted to be $W_{1,24}=10^{11} \mathrm{sec}^{-1}$. Now the K-conversion probabability for the EO transition to the $0^{+}$ground state i.s expressed by Church and $W$ eneser ${ }^{47}$ as $W_{K} / \rho^{2}=7 \times 10^{8} \mathrm{sec}^{-1}$ where $P$ as already defined is a measure of the strength of the EO matrix element. In Table 6, taken from Deutsch ${ }^{50}$ are listed the values of $\rho^{2}$ measured for several EO transitions $(0-0)$.

If the assumption is made that the $E \odot$ matrix element for $\mathrm{Zn} 68$ is not much different from that for Ge-70, we can take $\rho=0.11$, which is the value recently measured by Alburger 51 .

Now the conversion yield is expressed as 
Table 5. Summary of Results on Search for EO Transition

No. of cts. above 2. $1 \mathrm{Mev}$ with source in 105 hours $15 \pm 3.7$

No. of cts. above 2. $1 \mathrm{MeV}$ without source in 105 hours

$14 \pm 3.6$

Total no. of cts. due to conversion electrons if any: , in 10.5 hours

$$
\begin{aligned}
& =1 \pm 5 \\
& \sim 450 \mathrm{cps} \\
& \sim 450 \times 105 \times 3600
\end{aligned}
$$

Estimated no. of positrons in the beta spectrum

No. of disintegrations of positrons.

No. of disint egrations of $\mathrm{Ga}^{68} \sim \frac{450 \times 105 \times 3600}{1.9}$

$$
\text { assuming } \quad 0.90 \sim 1.9 \times 10^{8} \mathrm{cts} / 105 \mathrm{hr} \text {. }
$$

- 0 No. of conv. electrons per disintegration

$$
\sim \frac{1 \pm 5}{1.9 \times 10^{8}}(0.5 \pm 2.5) \times 10^{-8}
$$

or $(5 \pm 25) \times 10^{-9}$ per decay.

Table 6. Transition Probabilities for 0-0 Transitions

$\begin{array}{lll}\mathrm{C}^{12} & 7.68 & 0.06 \\ \mathrm{O}^{16} & 6.06 & 0.06 \\ \mathrm{Ge}^{70} & 1.21 & 0.015 \\ \mathrm{Ge}^{72} & 0.69 & 0.014 \\ \mathrm{Zr}^{90} & 1.75 & 0.036 \\ \mathrm{Pd}^{106} & 1.14 & 0.02 \\ \mathrm{Po}^{214} & 1.41 & 0.00257\end{array}$

Nucleus Transition
Energy, MeV 


$$
N_{K}=\frac{\left(w_{K} / \rho^{2}\right) \rho^{2}}{\left(\frac{W_{K}}{\rho^{2}}\right) P^{2}+W_{1.24}} \pm N
$$

where $\mathrm{N}$ is the total number of disintegrations and $f$ is the fraction of decays leading to the $2.3 \mathrm{MeV}$ level. From the data of Horen, $f$ is taken to be $10^{-3}$. Nf estimated from the positron spectrum is $2 \times 10^{5}$ in 105 hours. Substituting these numbers, the computed yield is $\mathrm{N}_{\mathrm{K}}=8.4 \times 10^{-9}$ conversion elec. trons per decay, to be compared with the measured value of $(5 \pm 25) \times 10^{-9}$.

If these estimates are indeed correct, then one can conclude that $0^{+}$ is an unlikely assignment for the 2. $3 \mathrm{MeV}$ level. This would give $\mathrm{rise}$ to the interesting possibility of the existence of a succession of $2^{+}$levels, if it is assumed on the basis of systematics ${ }^{52}$ of even-even nuclei in this region that the $1.88 \mathrm{MeV}$ level is $2^{+}$. The measured upper limit for the internal conversion electrons is indeed consistent with the expected yield of $<10^{-10}$ conversion electrons per decay for an E2 transition of 2. $3 \mathrm{MeV}$.

We have assumed rather tacitly that the single particle model description is valid in describing the gamma transition. It has been pointed out by Scharff-Goldhaber ${ }^{53}$ that the $1.24 \mathrm{MeV}$ gamma ray transition in Ge-70 is ten times faster than expected on the basis of the single particle model. If, by analogy with this, it turns out that the $1.24 \mathrm{MeV}$ gamma ray is equally fast. then our conclusions are somewhat weakened. It is therefore of obvious interest to perform a careful angular correlation of the $1.07-1.24 \mathrm{MeV}$ gamma cascade.

\section{Conclusions to Part $B$}

A search for a possible low-1ying $0^{+}$state in Ga-68 has been made through the decay of 270 day Ge-68 using NaI(TI) crystals. The results were negative. An upper limit of $10 / 0$ could be set on the gamma ray emission in Ge 68 decay. The failure to find the $0^{+}$state implies that the analogy of $\mathrm{Cu}$ 62 does not hold in this case. The absence of gamma rays in Ge-68 decay means that the levels in Ga-68 known from $\mathrm{Zn}-68$ (p, n)Ga-68 reaction must have spins of at least $\overline{2}$ if of even parity.

An upper limit of $0.40 / 0$ per decay could be set on the amount of positron emission by Ge-68. This is consistent with the decay proceeding purely by electron-capture as preditted by the beta energy systematics of Way and Wood 20 . 
A search for an electric monopole (EO) transition from the 2. $3 \mathrm{MeV}$ level in $\mathrm{Zn} .68$ to the ground state has been made, using plastic scintillators and a shielded anti-coincidence arrangement. Internal conversion electrons of $2.3 \mathrm{MeV}$ arising, from the $\mathrm{EO}$ modes of de-excitation were sought. An upper limit of $(5 \pm 25) \times 10^{-9}$ conversion electrons per decay of Ga-68 could be set. Assuming a value of 0.11 for $\rho$, the EO strength parameter, and the single particle model to apply in describing gamma transitions, the theoretically expected yield was $84 \times 10^{-9}$ conversion electrons per decay. The mea. sured upper limit is interpreted as evidence against a possible $0^{+}$assignment for the $2.3 \mathrm{MeV}$ level, but the interpretation. must be viewed with reservation owing to the assumptions made in computing the theoretical yield. It. is sug gested that an angular correlation of the 1.07. MeV - 1.24 MeV gamma cascade be made with a strong source to decide between the $0^{+}$and $2^{+}$spin as. signments. 


\section{References}

1. Hermannsfeldt, Stahelin. Maxson, and Allen, Phys. Rev. 107, 641 (1957)

Hermannsfeldt, Burman, Stahelin, Allen, and Braid, Phys. Rev. Lett。

$1,61(1958)$

W. P. Alford and D. R. Hamilton, Phys. Rev。95, 1351 (1954)

Burman, Hermannsfeldt, Allen, and Braid, Phys. Rev。 Lett. 2, 9 (1959)

2. M. Fierz, Z. Physik, 10.4, 553. (1937)

3. J. B. Gerhart, Phys. Rev. 109, 897 (1958)

4. H. Daniel and U. Schmidt-Rohr, Nuclear Physics 7, 516 (1958)

5. A. Altman and.W. M. MacDonald, Phys.Rev. Lett. 1, 456 (1958)

6. R. Sherr and R. H. Miller, Phys. Rev. 93, 1076 (1954)

7. P.F. Zweifel, Proceedings of Rehovoth Conference (North-Holland Pub.lishing Company 1957). However, recent work of Konijn et al. has indicated that this may not be true: see reference 38 .

8. H. Brysk and M. E. Ro.se, Rev. Mod. Phys. 30, 1169 (1958)

9. Perl ${ }_{n} W_{\text {W }}$ elker and.Wolfsberg, Phys. Rev. 110, 381 (1958)

10. M.E. Rose and J. Jackson, Phys. Rev. 76, 1549 (1949)

11. A. M. Mukerji and P. Preiswerk, Helv. Phys.Acta, 25, 387 (1952)

12. Crasemann, Rehfuss and Easterday, Phys.Rev. 102, 1344 (1956)

13. H. Daniel, Z. Naturf. 12a, 363 (1957)

14. G. Scharff-Goldhaber, Phys. Rev.90, 387 (195.3)

15. Hubbs, Marrus and.Worcester, Phys. Rev. 110, 534 (1958)

16. P. M. Endt and C. M. Braams, Rev. Mod. Phys. 29, 683 (1957)

17. P. R. Bell. in Beta and Gamma-Ray Spectroscopy: edited by K. Siegbahn (North-Holland Publishing Company, 1955) p. 154

18. D. J. Horen, Phys. Rev. 113, 572 (1959) and private communication; June 1958

19. M. E. Rose in Beta and Gamma-Ray Spectroscopy: edited by K. Siegbahn (North-Holland Publishing Company, 1955), appendix 4

20. K. Way and M. Wood, Phys. Rev. 94, 119 (1954)

21. C. M. Davisson in Beta and Gamma-Ray Spectroscopy, edited by K. Siegbahn (North-Holland Publishing Company, 1955), p. 124

22. R. A. Chapman and J. C. Slattery, Phys. Rev. 105, 633 (1957)

23. R. M. Sinclair, Westinghouse Research Report 60, 94511, 6. (1956) p. 12

24. Van Patter, Rothman, and Mandeville, Phys. Rev. 107, 71 (1957) 
25. Tables for the Analysis of Beta-Spectra, National Bureau of Standards Applied Mathematics Series No. 13 (U.S. Covt. Printing Office, Washington, D. C. 195.2)

26. M. K. Ramaswamy, Bull. Am. Phys. Soc. 2, 4, 151. (1959)

27. M.K. Ramaswamy, Nuclear Physics, 10, 205 (1959)

28. Frauenfelder, LeVine, Rossi and Singer, Phys. Rev。103, 352 (1956)

29. R. W. King, Rev. Mod. Phys. 26, 327 (1954)

30. W. Dobrov and C. D. Jeffries; Phys. Rev. 108, 60. (1957)

31. Dagley, Grace, Hill and Sowter, Phil. Mag. 3, 489 (1958)

32. Good, Peaslee and Deutsch, Phys. Rev. 69, 313 (1946)

33. C. S. Cook and F. Tomnovec, Phys. Rev. Bull. Am. Phys. Soc. 1, 5, 253 (1956)

34. M. K. Ramaswamy, Bull. Am. Phys. Soc. 2, 3, 357 (1958)

35. Konijn, Van Nooijen; Van Krugten and Sobben, Physica 24, 931 (1958)

36. J.E. Mack, Rev. Mod. Phys. 22, 64 (1950)

37. Hinman, Bower and Leamer, Phys. Rev. 90, 370 (1953)

38. Konijn, Van Nooijen; Hagedoorn and Wapstra, Nuclear Physics 9 , 295 (1959)

39. Johnson, Johnson and Langer, Phys. Rev. 102, 1142 (1956)

40. R. L. Robinson and L. M. Langer, Phys. Rev. 109, 1255 (1958)

41. M. K. Ramaswamy, submitted to Current Science

42. Hubbs, Nierenberg, Shugart and Worcester, Phys. Rev. 105, 1928 (1957)

43. Way, Kundu, McGinnis and van Lieshout, Ann. Revs. Nuclear Science 6. 129 (1956)

44. Way, King, McGinnis and van Lieshout, Nuclear Level Schemes A 40.92 (U.S. Govt. Printing Office, Washington, D.C. 1955)

45. E. J. Swift, J. Am. Chem. Soc. 46, 2375 (1924)

46. R. G. Thomas, Phys. Rev. 58, 714 (1940)

47. E. L. Church and J. Weneser, Phys. Rev, 103, 1035 (1956)

48. D. R. Tilley, Ph. D. Dissertation, Johns Hopkins University 1958 (unpublished)

49. S. A. Moszkow ski in Beta and Gamma-Ray Spectroscopy: edited by K. Siegbahn (North-Holland Publishing Co. 195.5) p. 373 
50. M. Deutsch, Nuclear. Physics 3, 83 (1957)

51. D. E. Alburger, Phys. Rev. 109, 12.22 (1.958)

52. D. M. Van Patter, Bull. Am. Phys. Soc. 2; 3, 212 (1958)

53. G. Scharff $\sim$ Goldhaber, private communication to Professor L. Madansky (January 1959) 
1. Ga ${ }^{68}$ decay scheme as given by Crasemann et al.

2. Schematic of counter set-up for $\mathrm{K} / \beta+$ measurements

3. Singles gamma ray spectrum $(0.3$ to $1.2 \mathrm{MeV})$ of $\mathrm{Ga}^{68}$ in $2^{\prime \prime}$ cube $\mathrm{NaI}(\mathrm{Tl})$ crystal

4. Decay scheme of $\mathrm{Co}^{58}$ according to Frauenfelder et al

5. Typical singles gamma spectrum of $\mathrm{Co}^{58,60}$ taken in $2^{\prime \prime}$ cube $\mathrm{NaI}(\mathrm{Tl})$ crystal. The dotted curve is the spectrum of $\mathrm{Co}^{60}$ only normalized to $\mathrm{Co}^{58,60}$ spectrum

6. $0.511-0.810 \mathrm{Mev}$ coincidences in $\mathrm{Co}^{58}$

7. Beta spectrum of $\mathrm{N}^{13}$ in $\mathrm{NaI}(\mathrm{Tl})$ sandwich. Also shown is the energy calibration using external gamma sources.

8. Fermi plot of $\mathrm{N}^{13}$ beta spectrum showing the end-point at $1.16 \mathrm{MeV}$

9. a) Portion of beta spectrum of $\mathrm{N}^{13}$ and (b) the beta spectrum coincident with annihilation radiation

10. Decay.scheme of $\mathrm{Na}^{22}$

11. Fermi plot of $\mathrm{P}^{32}$ spectrum taken in $4 \pi$ plastic scintillator. Note that the end-point occurs at $1.72 \mathrm{MeV}$

12. a) dashed line, beta spectrum of $\mathrm{Na}^{22}$ in $4 \pi$ plastic scintillator, in coincidence with $1.28 \mathrm{MeV}$ gamma ray

b) dotted curve: Beta spectrum of $\mathrm{Na}^{22}$ in triple coincidence with the twp annihilation quanta and $1.28 \mathrm{MeV}$ gamma ray normalized to the doubles spectrum (beta - 1.28) above $50 \mathrm{keV}$

c) Energy calibration of the $4 \pi$ scintillator with Compton edges of internal gamma sources of $\mathrm{Na}^{22}(0.511 \mathrm{MeV})$ and $\mathrm{Cs}^{137}(0.661 \mathrm{MeV})$

d) Compton distribution of $\mathrm{Na}^{22}$ gamma rays in $4 \pi$ plastic scintillator (with positrons stopped in just enough lucite) coincident with annihilation radiation and $1.28 \mathrm{MeV}$ gamma ray

13. Block diagram of experimental set-up for $\mathrm{Na}^{22}$ experiments

14. Study of systematic errors in $\mathrm{Na}^{22}$ experiment. Plot of h!/h as a function of cut-off energy $h_{\text {min }}$. The dashed line corresponds to ideal Fermi distribution distorted for resolution

15. Curve a. Low energy ( 0 to $30 \mathrm{keV}$ ) gamma spectrum in thin $\mathrm{NaI}(\mathrm{Tl})$ crystal taken with $\mathrm{Ge}^{68}+\mathrm{Ga}^{68}$ in equilibrium 
15. Curve b. Pulse height spectrum of the same energy taken immediately after chemical separation of $\mathrm{Ga}^{68}$ from Ge $\mathrm{fr}^{68}$

Gurve c. Pulse height spectium $(0-30 \mathrm{keV})$ coincident with $K \mathbf{X}+\mathrm{ray}$

16. Decay scheme of $\mathrm{Ga}^{68}$ as given by Horen.

17. Block diagram of anti coincidence arrangement used in the search for EO transition in $\mathrm{Zn}^{68}$. Shielding not shown

18. Positron spectrum of $\mathrm{Ga}^{68}$ observed in the anti-coincidence system: Also shown for reference is the beta-spectrum of $\mathrm{P}^{32}$ (singles) 
Fg 1

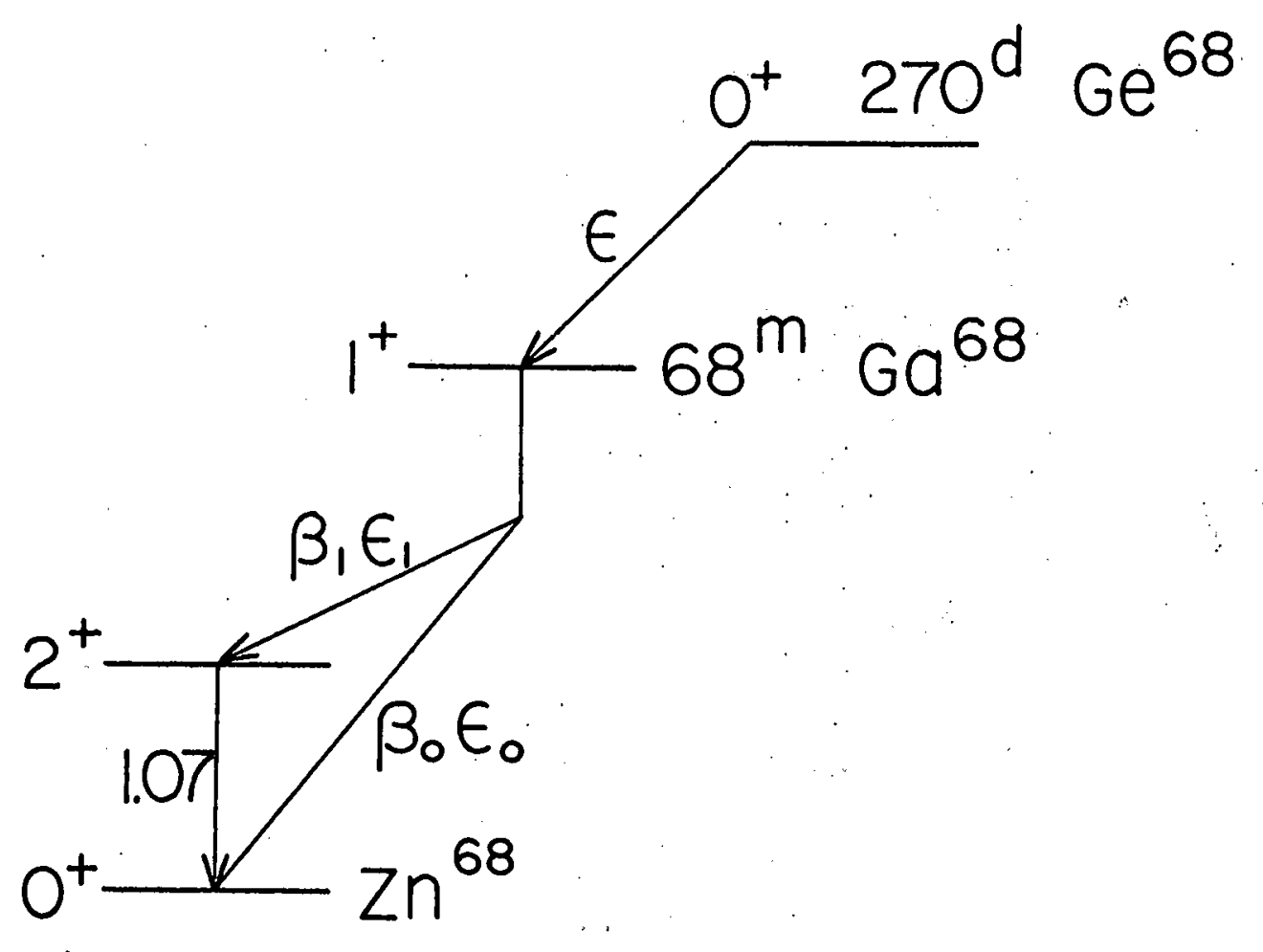

Stable

b 
2

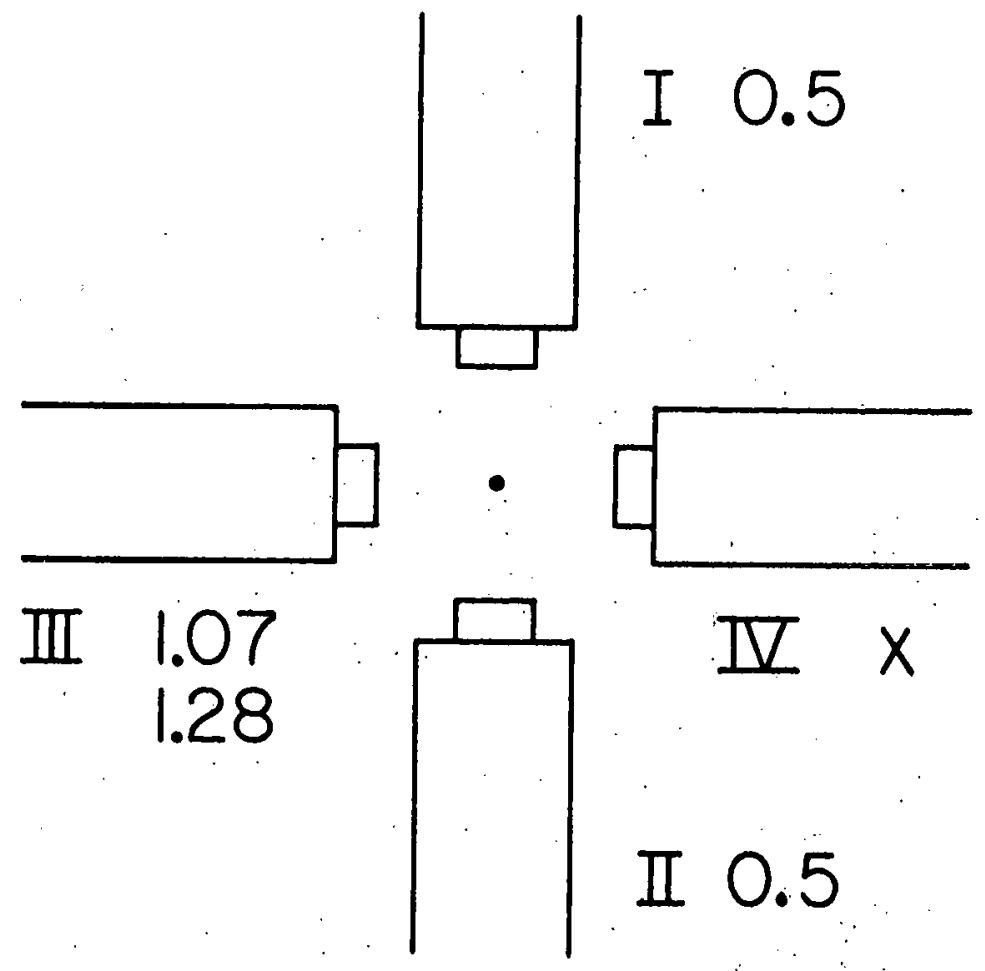

a 


\section{3}

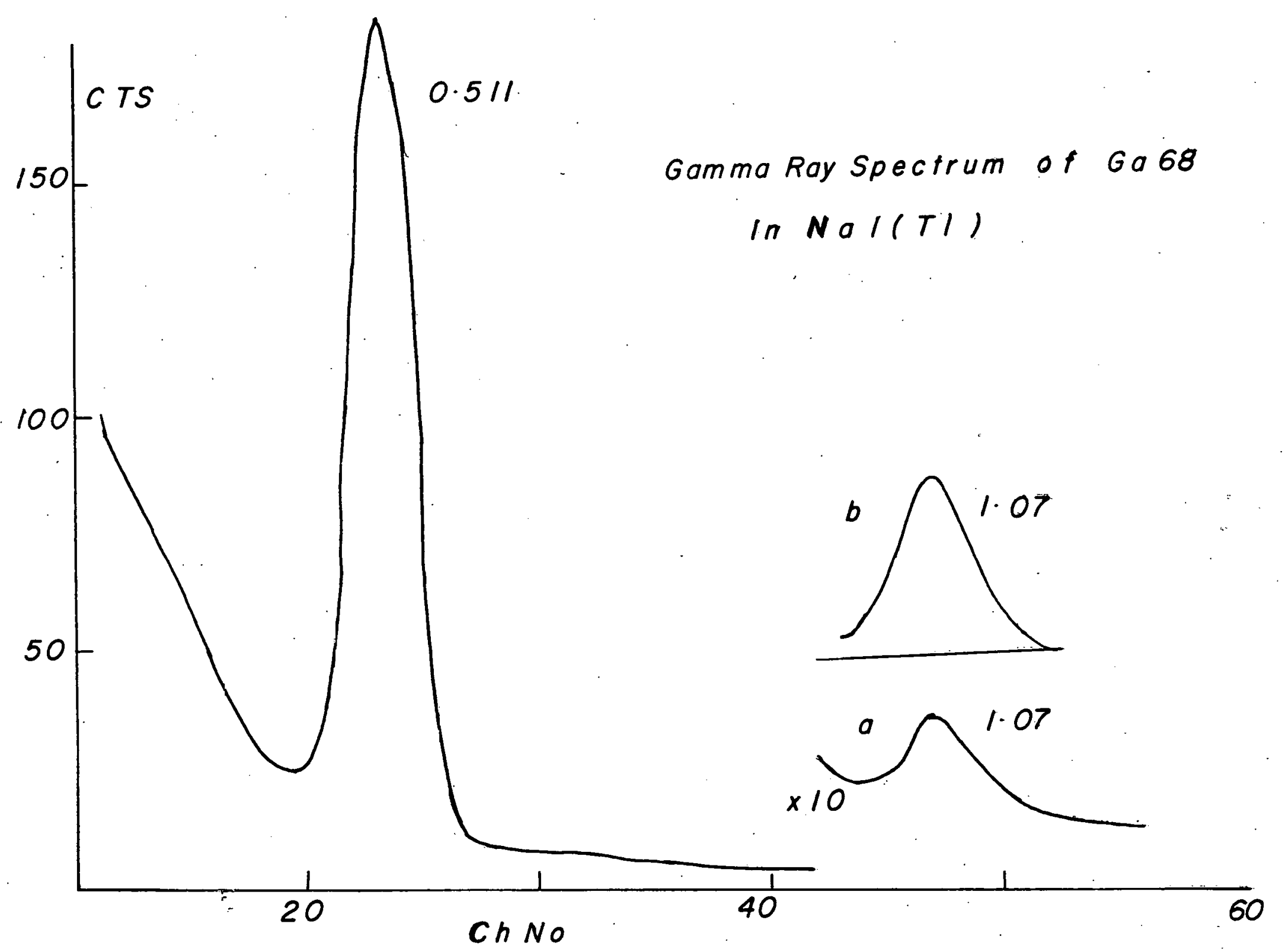


Decay Scheme of Co :58

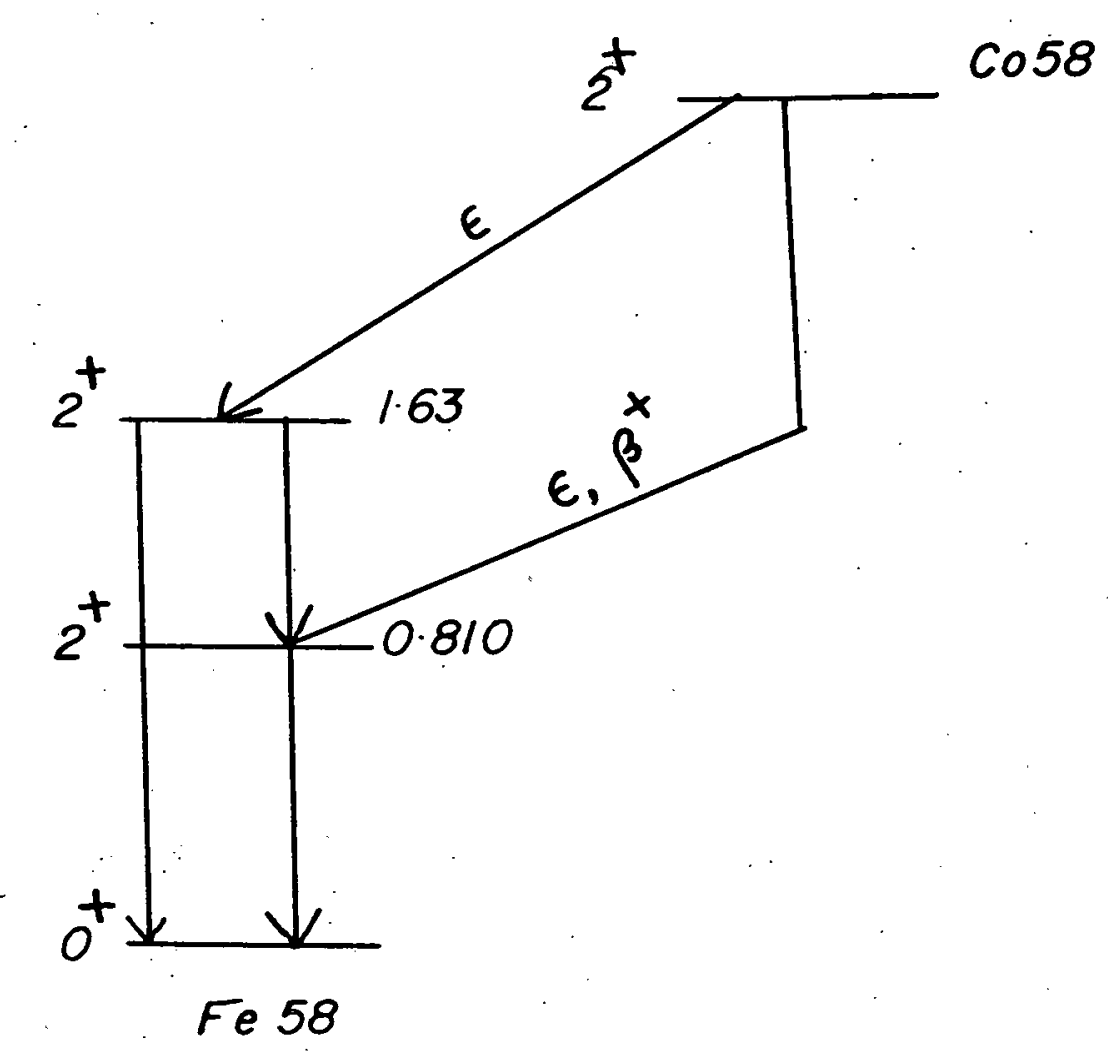

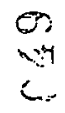

$\infty$ 


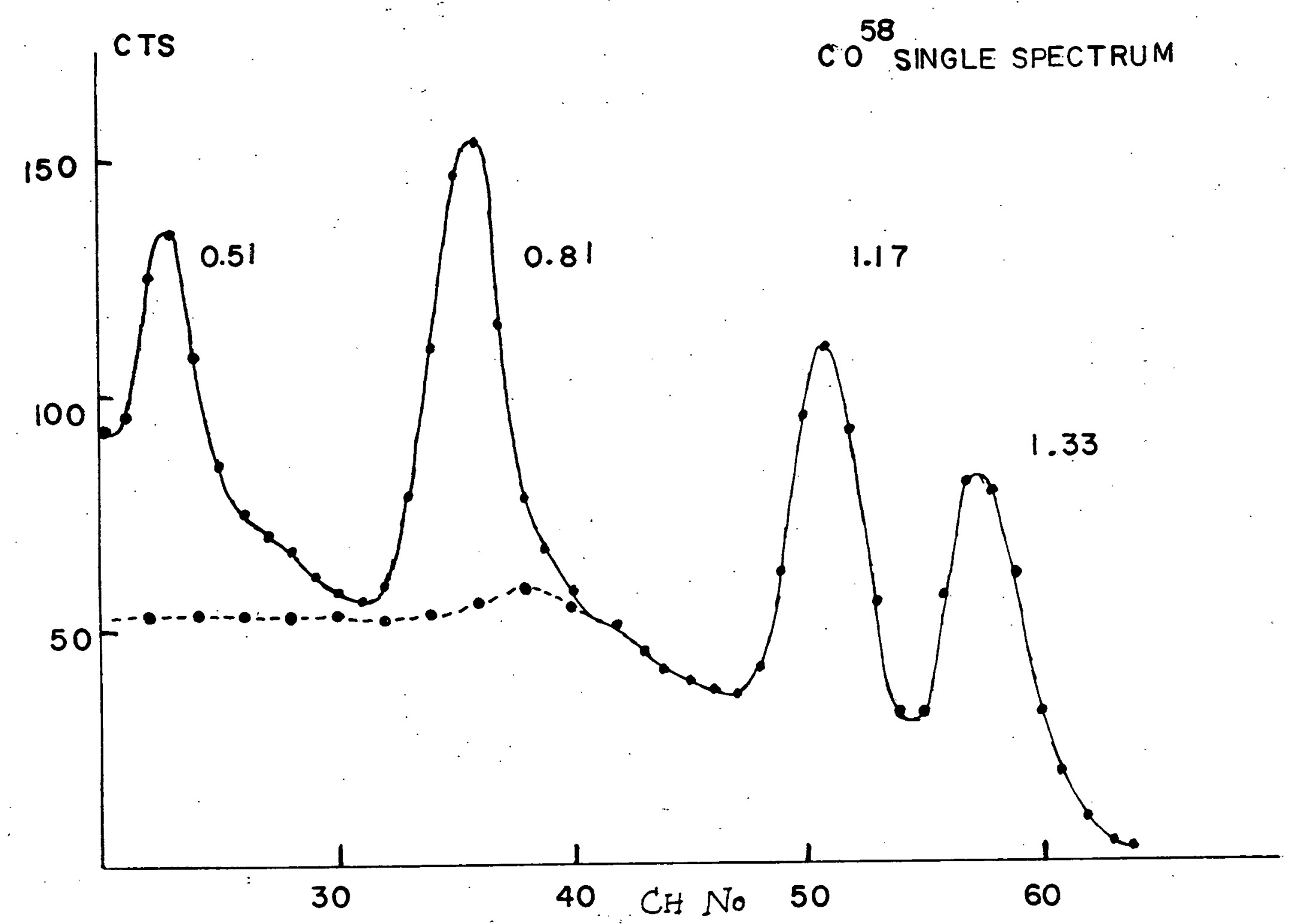


6

CTS

$$
\begin{array}{ll}
\mathrm{CO}^{58} & 0.5-0.8 \\
& \text { C OINCID ENCES }
\end{array}
$$

ind

$\frac{5}{0}$ 


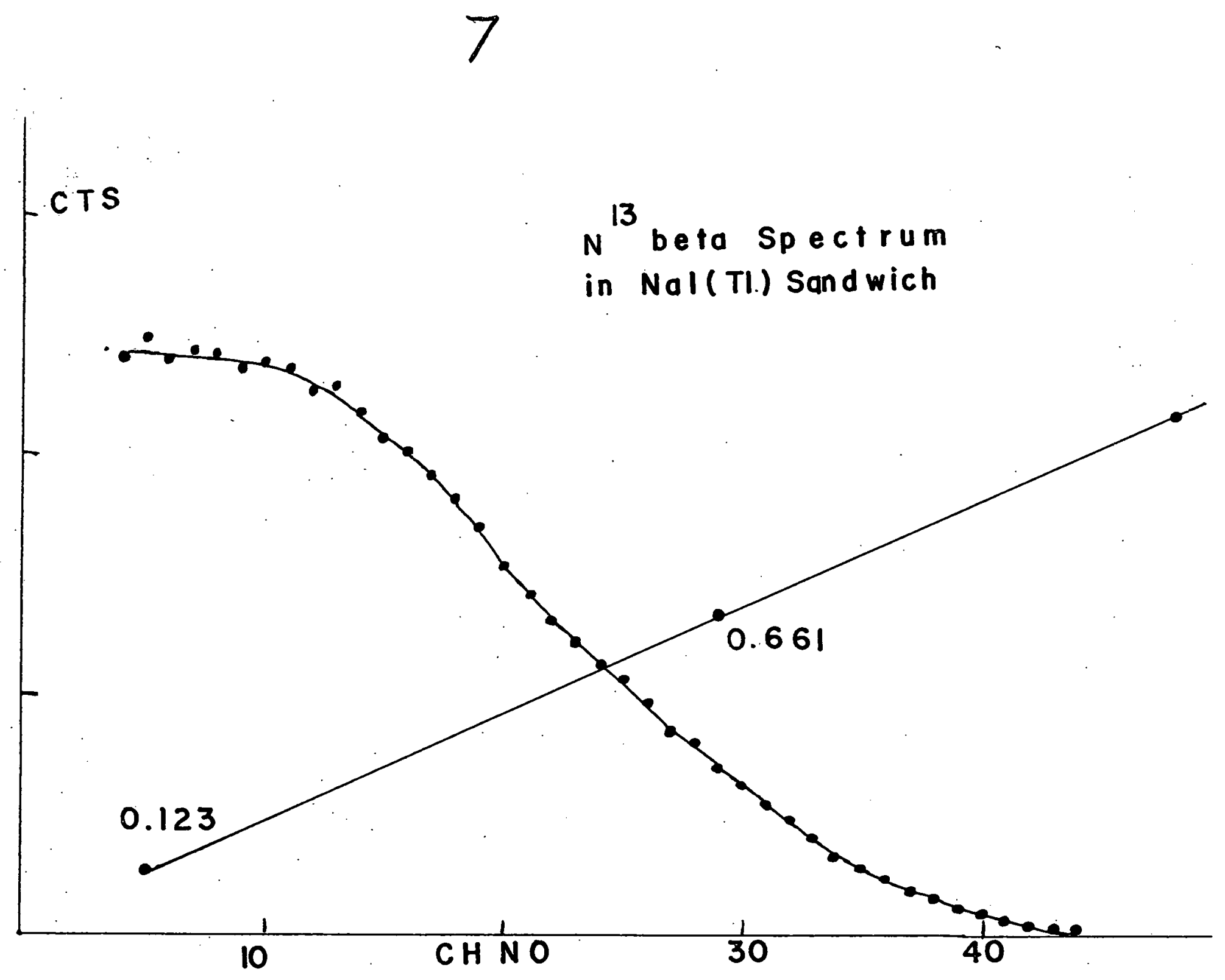




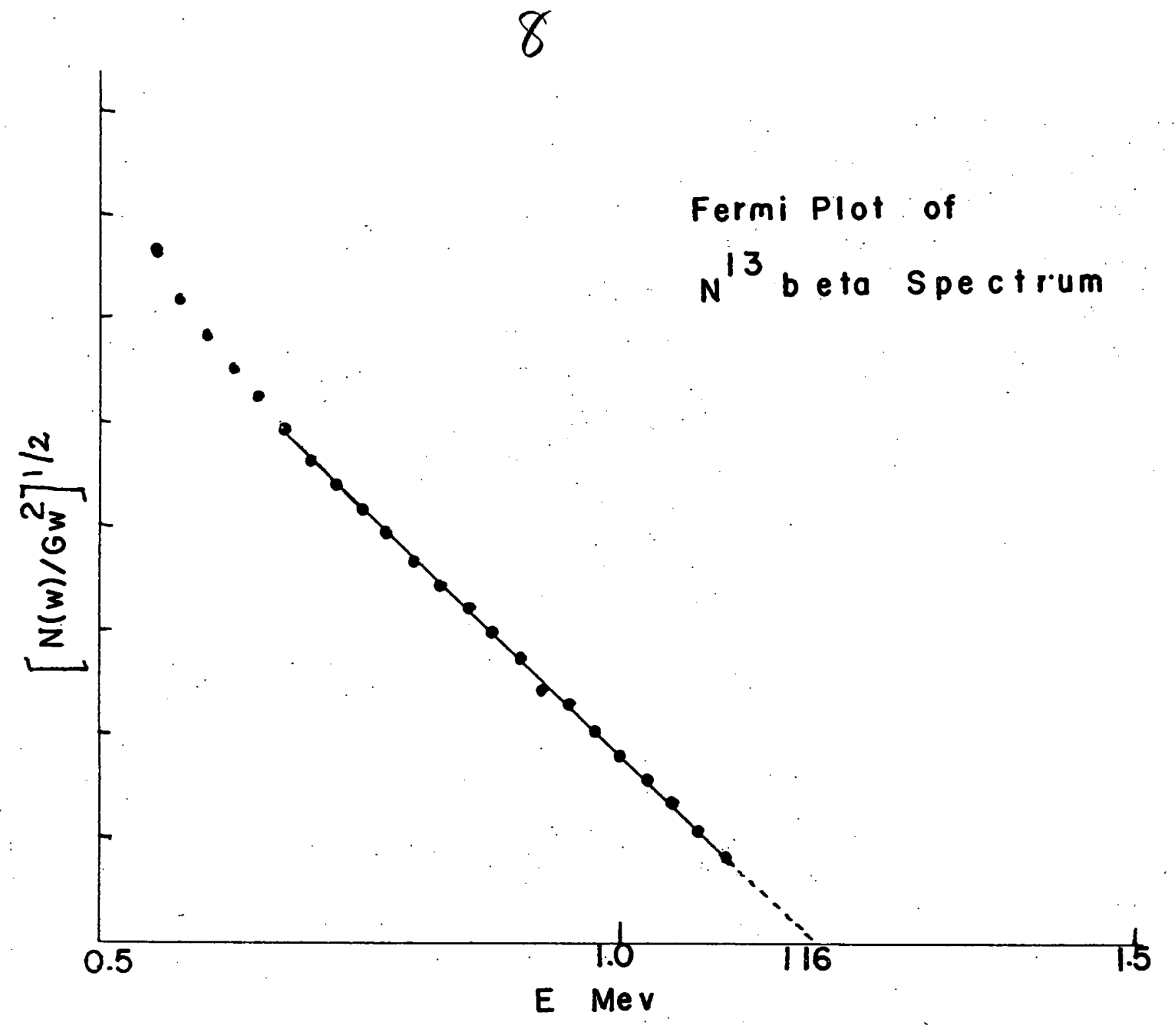


9

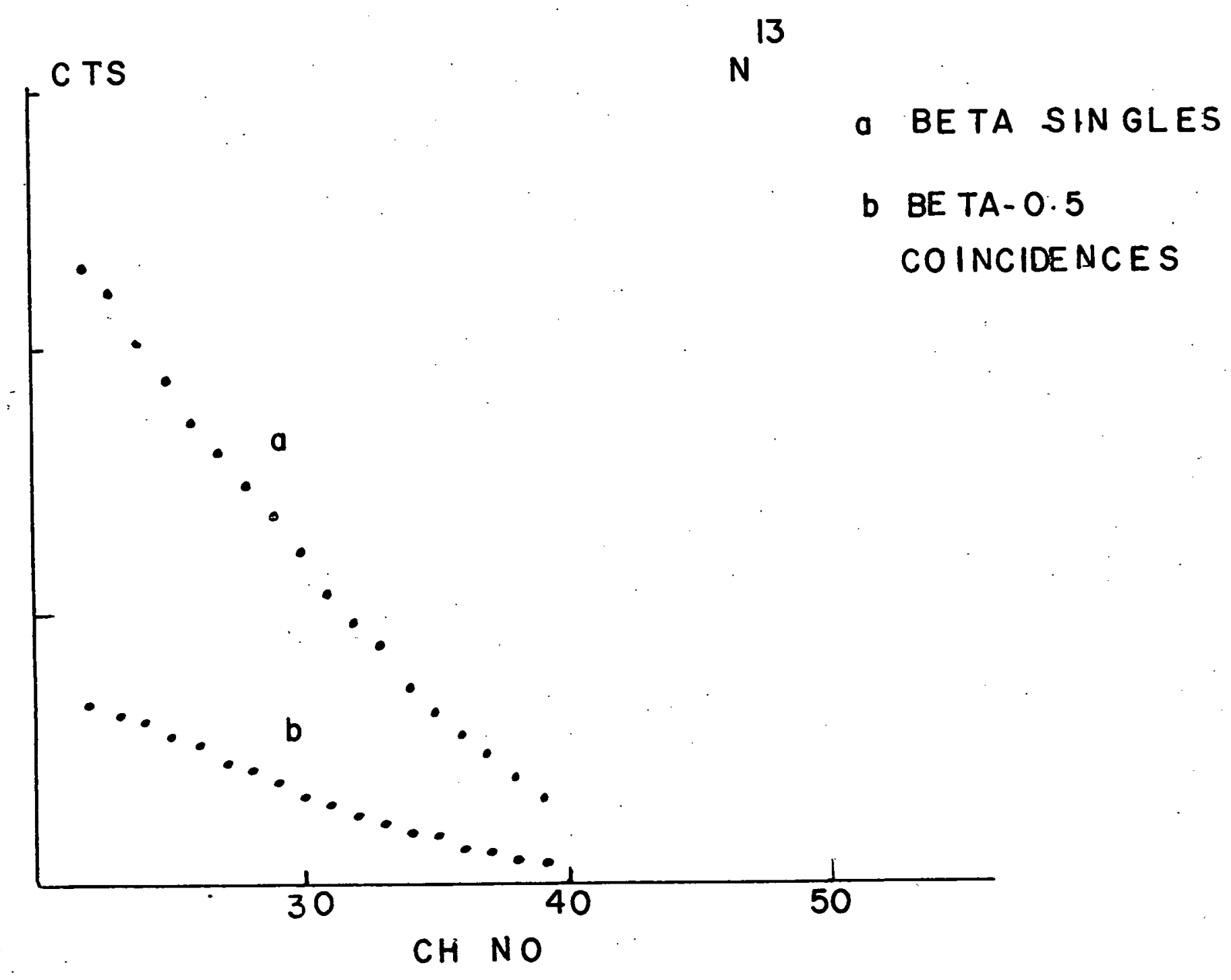




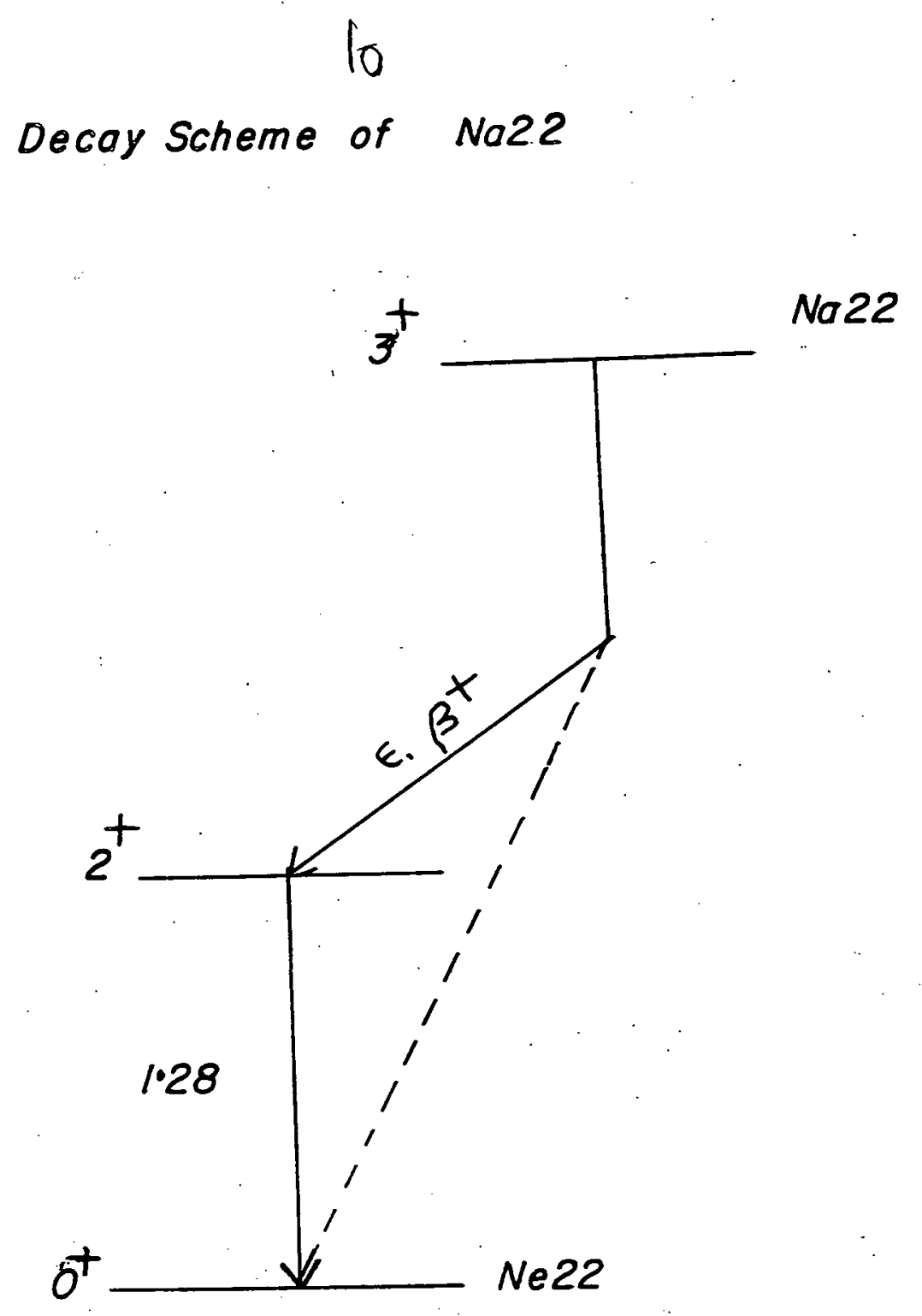




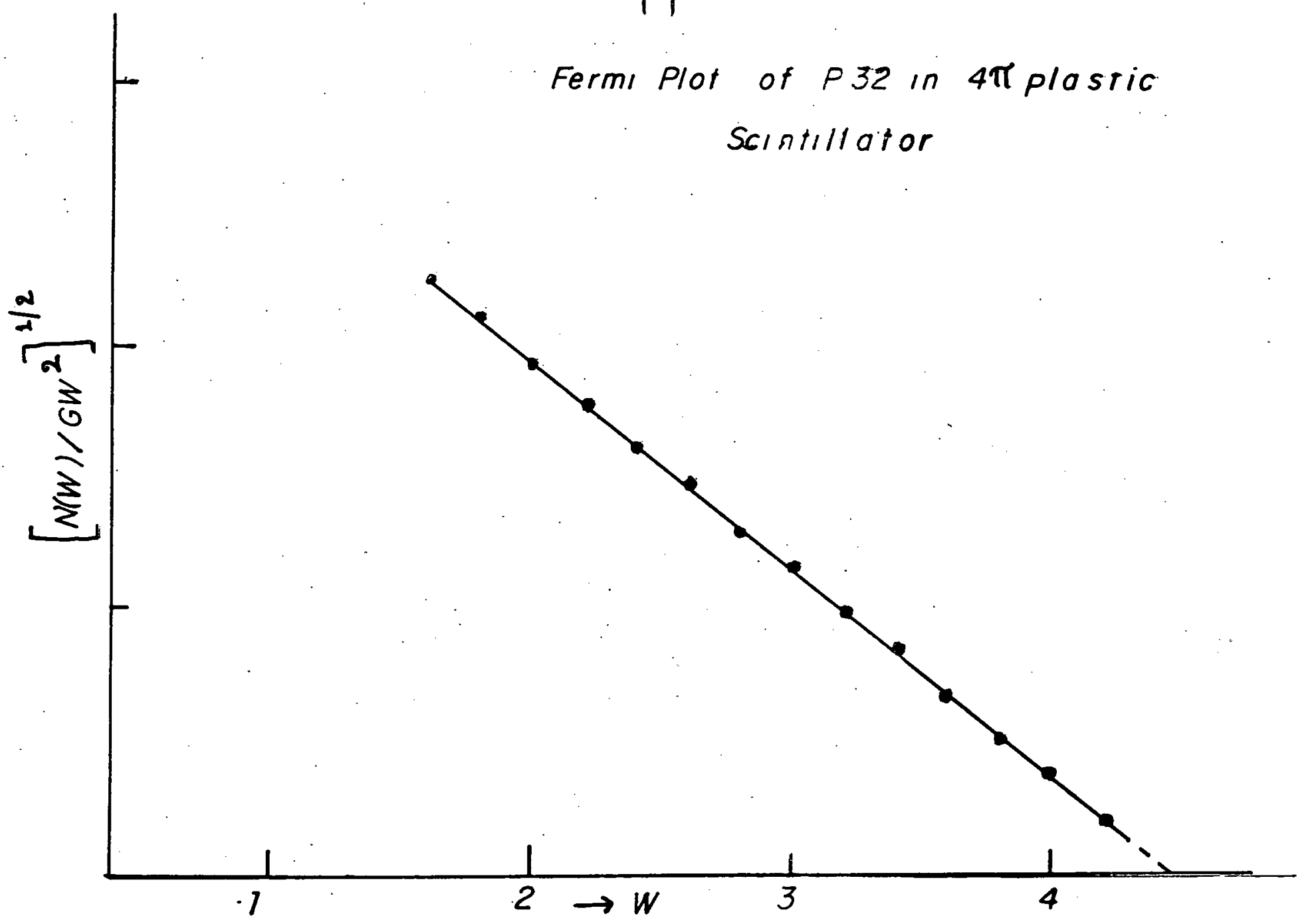




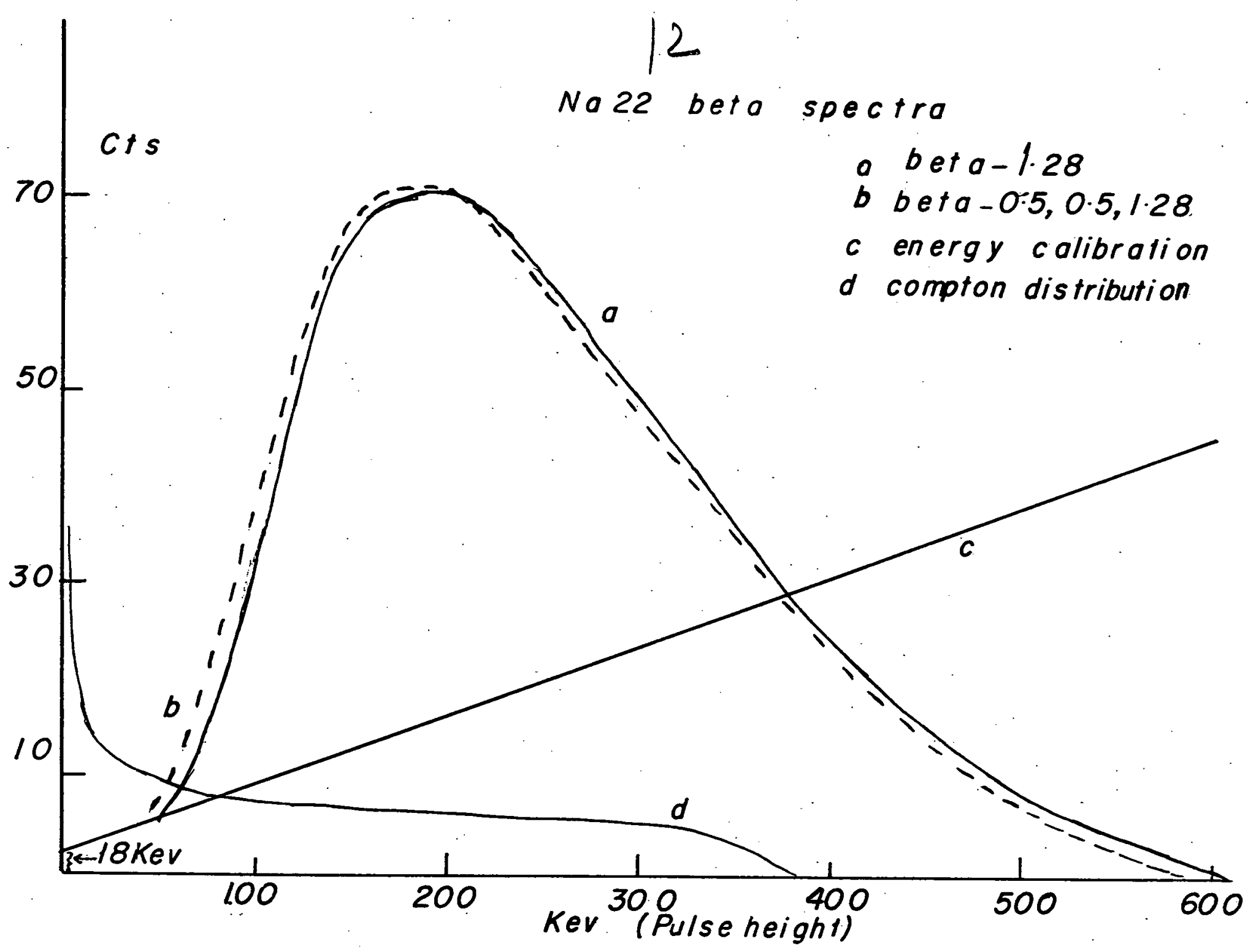


13

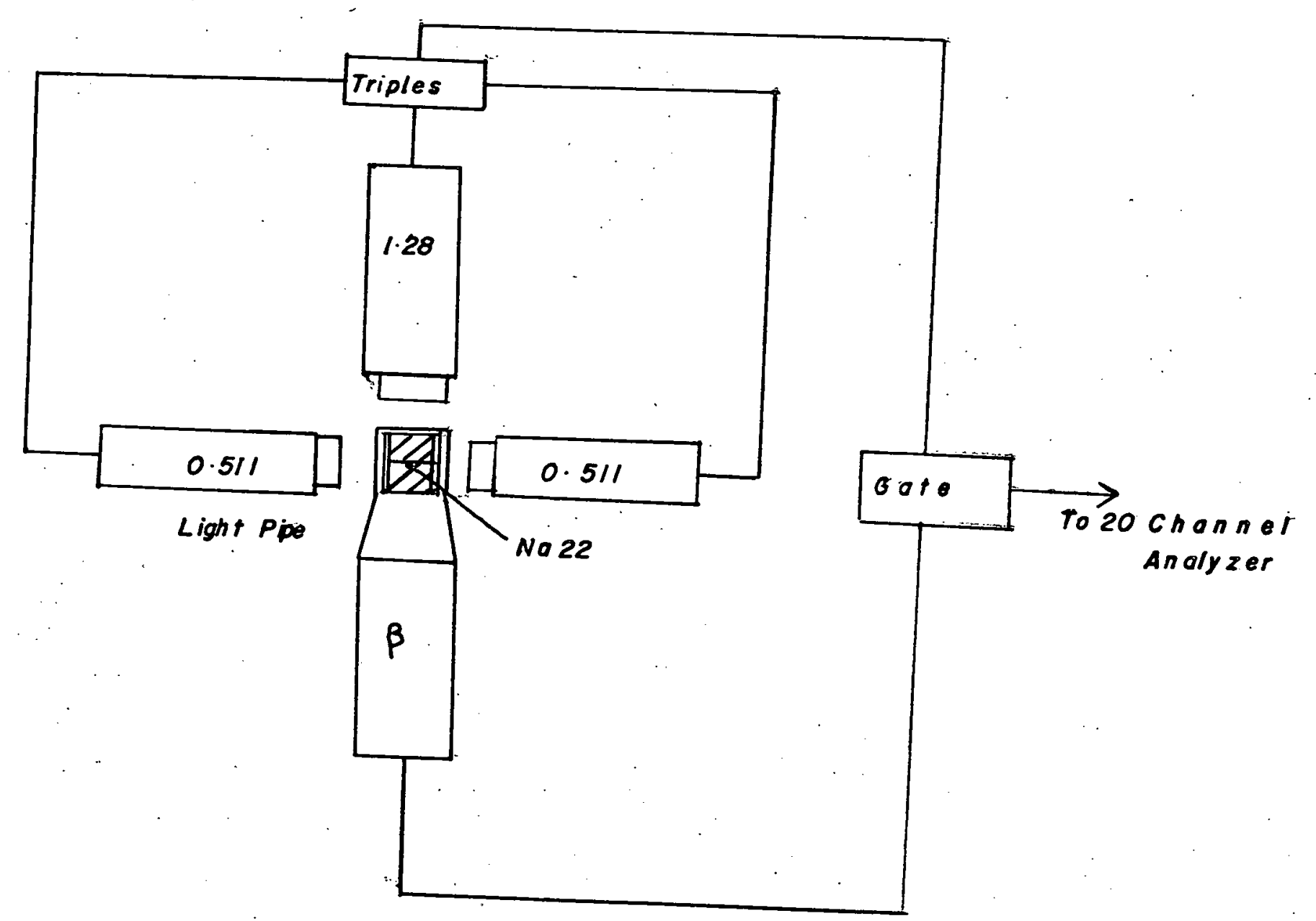




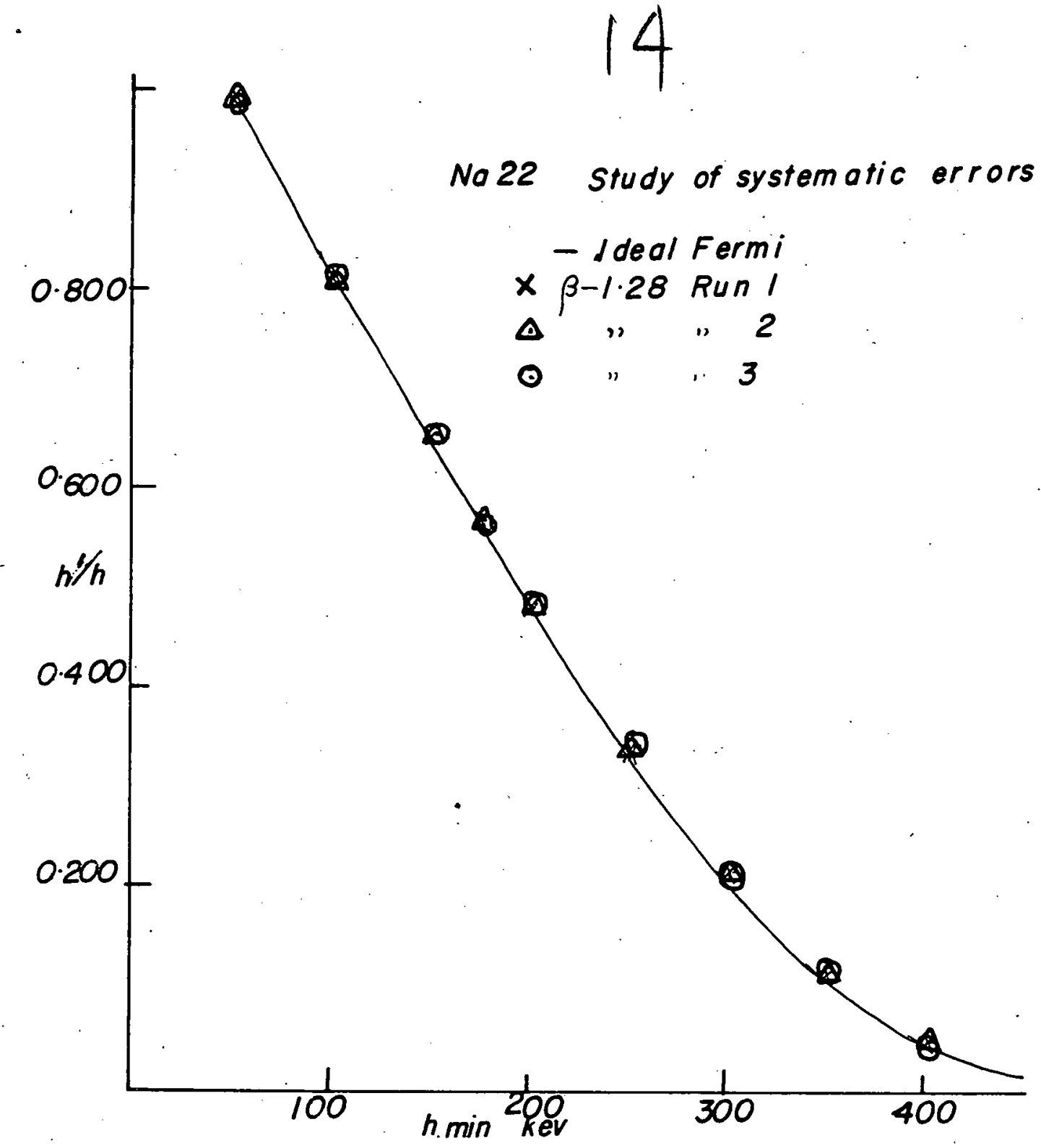




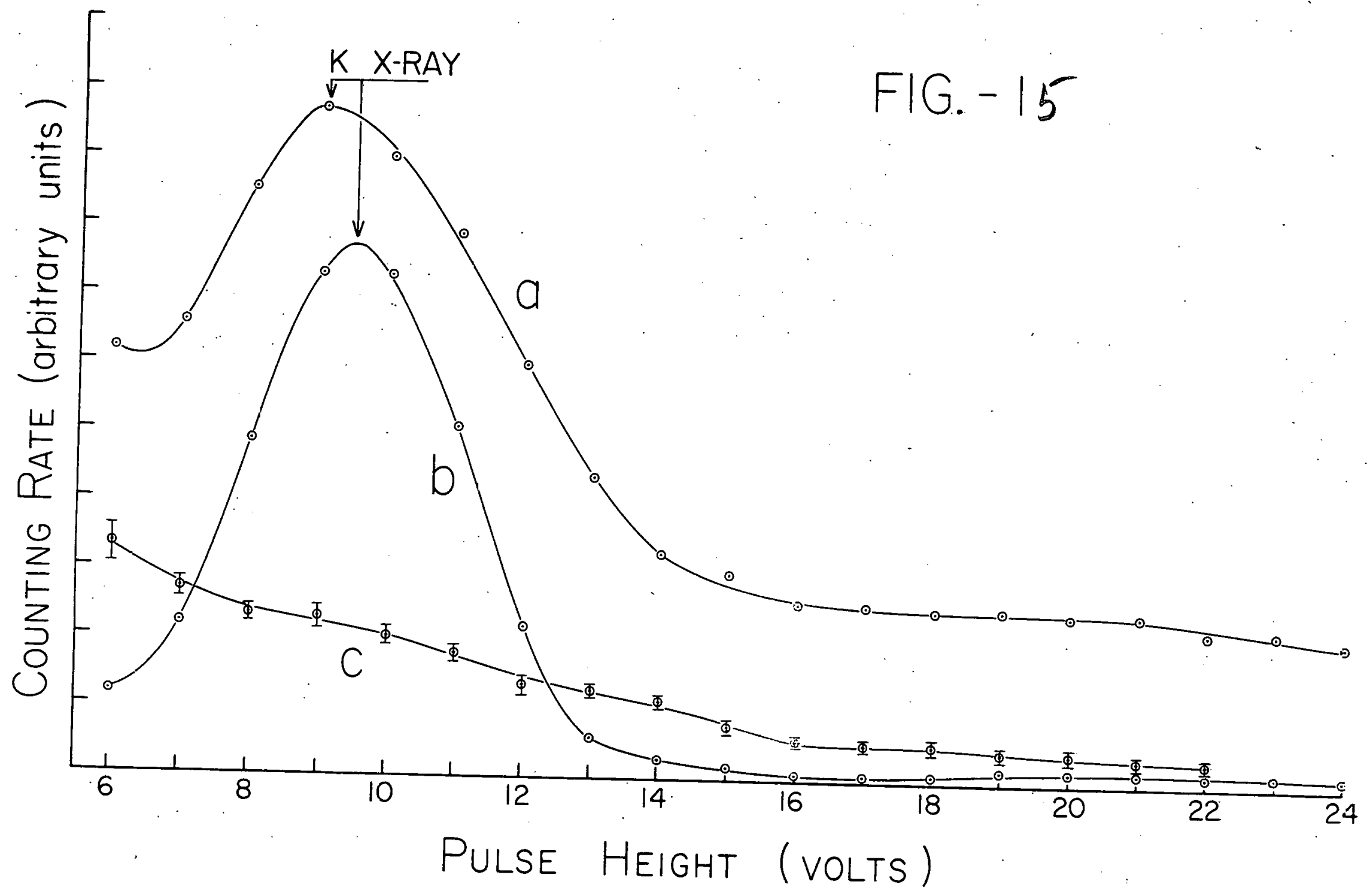


16

8

Decay Scheme of 6 a 68 according to Horn

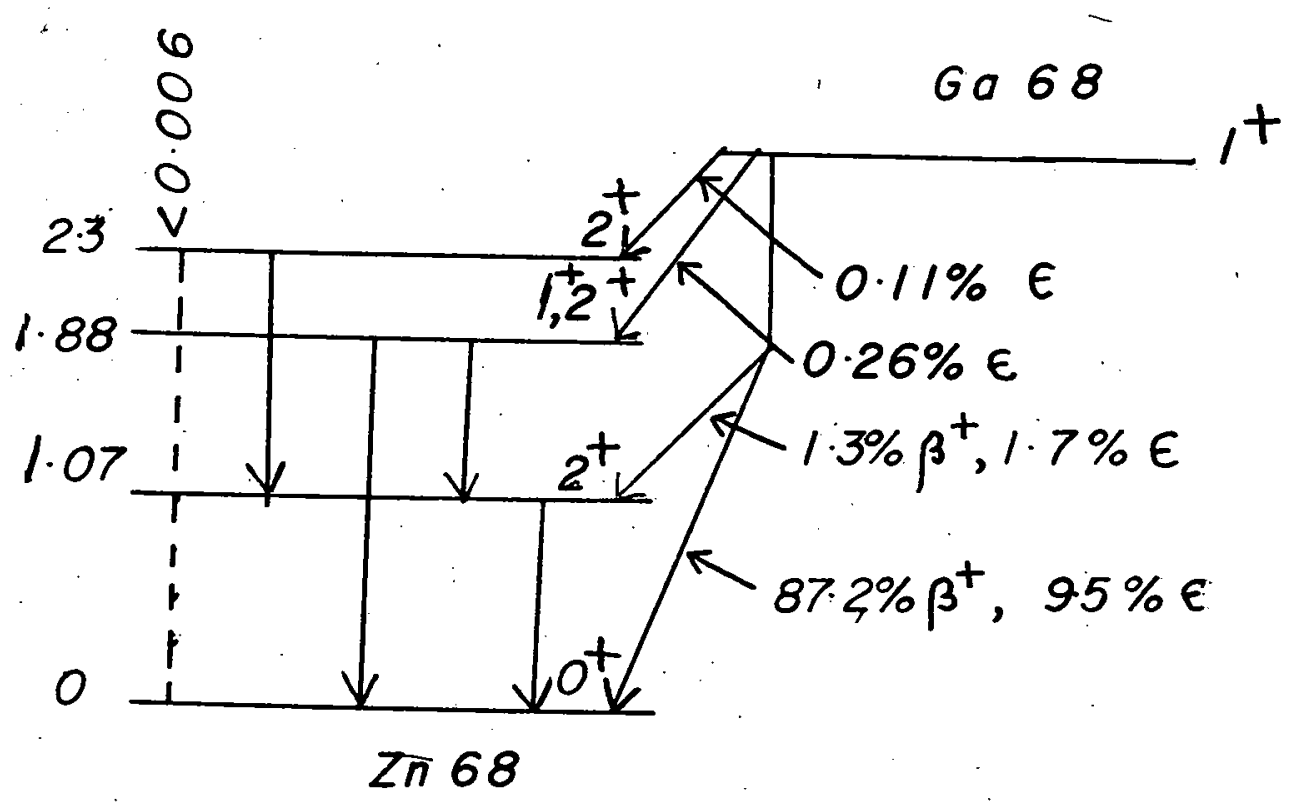

8 


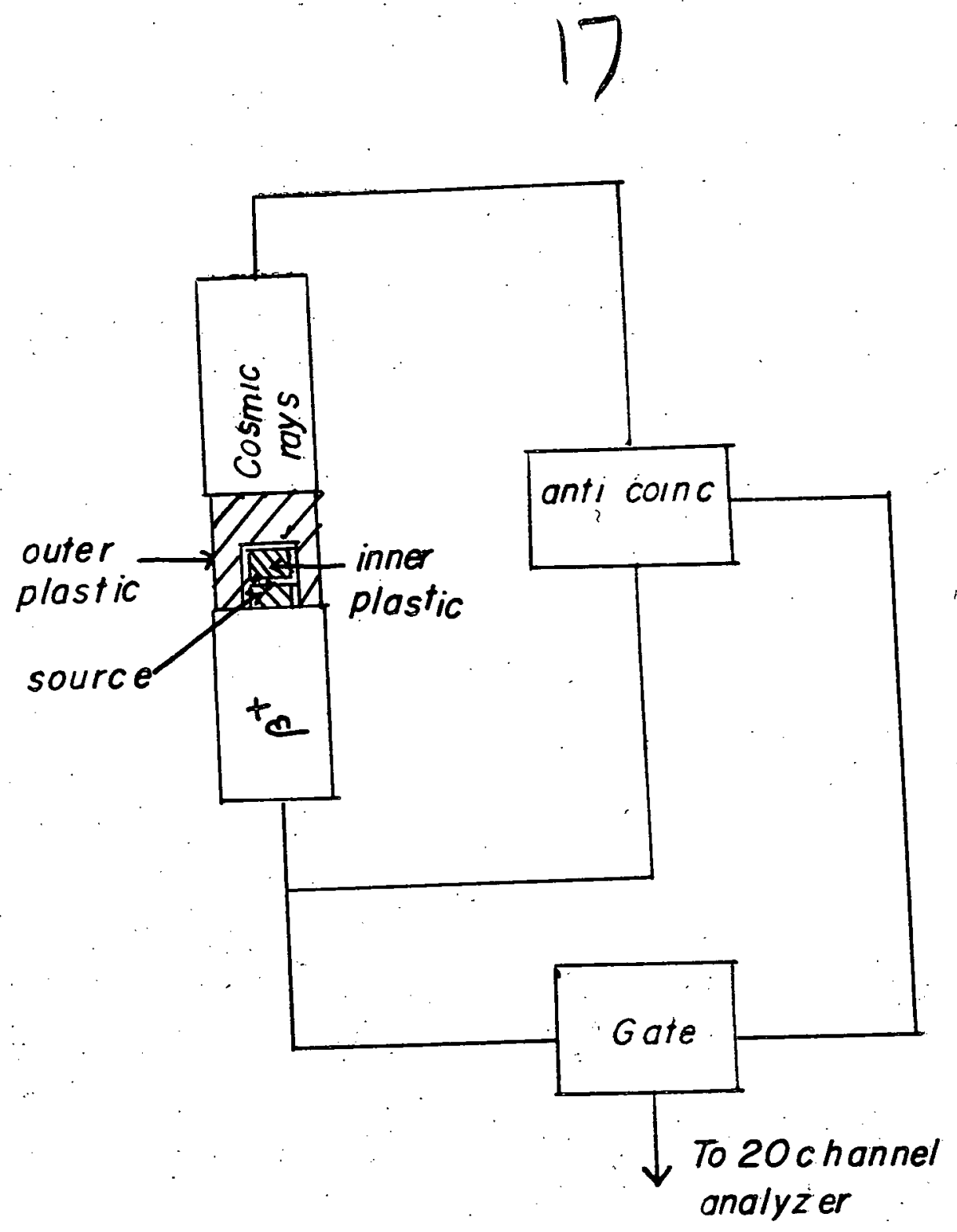

ges

0

5

0 


\section{8}

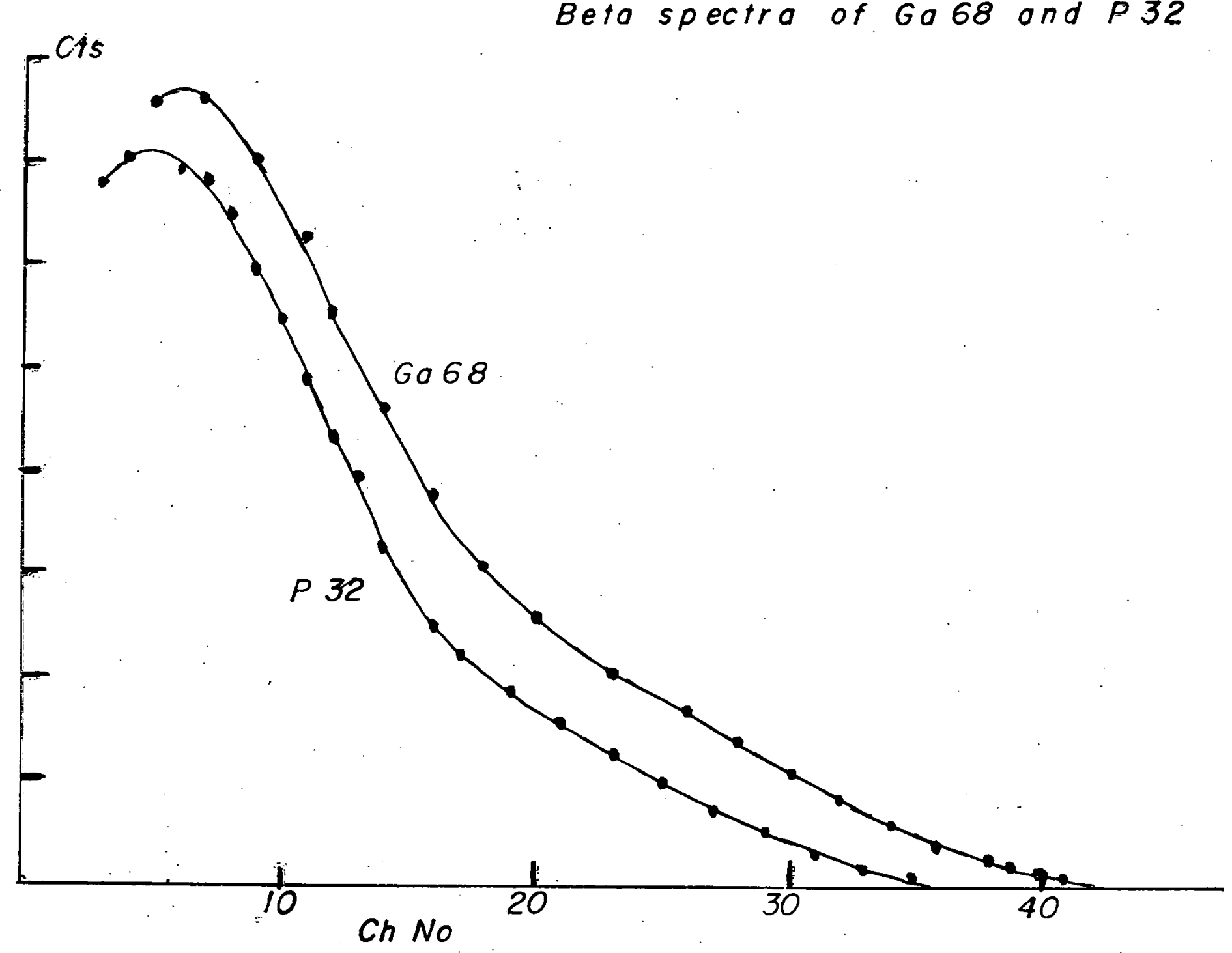

